Supporting Information

\title{
Can Primary Arylamines Form Enamine? Evidence, $\alpha$-Enaminone, and [3+3] Cycloaddition Reaction
}

E. H. Nisala Fernando, ${ }^{\text {a }}$ Jose Cortes Vazquez, ${ }^{\mathrm{a}}$ Jacqkis Davis, ${ }^{\mathrm{a}}$ Weiwei Luo, ${ }^{\mathrm{b}}$ Vladimir N. Nesterov, ${ }^{\mathrm{a}}$ and Hong Wang*a

${ }^{a}$ Department of Chemistry, University of North Texas, Denton, TX 76203, USA.

${ }^{\mathrm{b}}$ School of Chemistry and Food Engineering, University of Science and Technology, Changsha 410114, China

${ }^{\xi}$ Deceased 04/21

Table of Contents

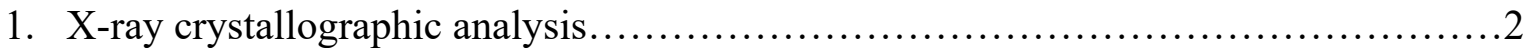

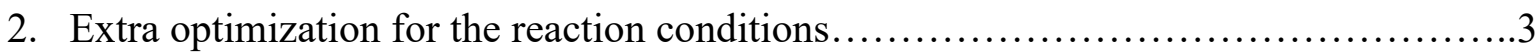

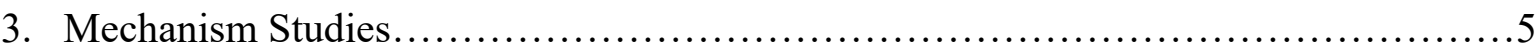

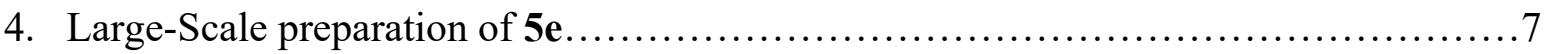

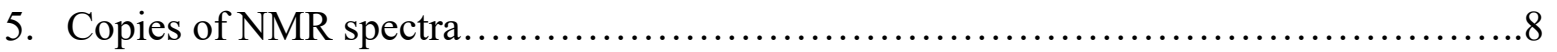




\section{X-ray crystallographic analysis}

Table S1. Crystal data and structure refinement for $3 \mathrm{i}$

\begin{tabular}{|c|c|c|}
\hline Identification code & \multicolumn{2}{|l|}{$3 \mathbf{i}$} \\
\hline Empirical formula & \multicolumn{2}{|l|}{ C18 H17 N O } \\
\hline Formula weight & \multicolumn{2}{|l|}{263.32} \\
\hline Temperature & \multicolumn{2}{|l|}{$99.98(11) \mathrm{K}$} \\
\hline Wavelength & \multicolumn{2}{|l|}{$1.54184 \AA$} \\
\hline Crystal system & \multicolumn{2}{|l|}{ Orthorhombic } \\
\hline Space group & \multicolumn{2}{|l|}{ Pbca } \\
\hline \multirow[t]{3}{*}{ Unit cell dimensions } & $\mathrm{a}=7.31130(10) \AA$ & $\alpha=90^{\circ}$. \\
\hline & $\mathrm{b}=6.92370(10) \AA$ & $\beta=90^{\circ}$. \\
\hline & $c=54.4177(7) \AA$ & $\gamma=90^{\circ}$ \\
\hline Volume & \multicolumn{2}{|l|}{$2754.69(7) \AA^{3}$} \\
\hline Z & \multicolumn{2}{|l|}{8} \\
\hline Density (calculated) & \multicolumn{2}{|l|}{$1.270 \mathrm{Mg} / \mathrm{m}^{3}$} \\
\hline Absorption coefficient & \multicolumn{2}{|l|}{$0.612 \mathrm{~mm}^{-1}$} \\
\hline $\mathrm{F}(000)$ & \multicolumn{2}{|l|}{1120} \\
\hline Crystal size & \multicolumn{2}{|c|}{$0.1 \times 0.05 \times 0.035 \mathrm{~mm}^{3}$} \\
\hline Theta range for data collection & \multicolumn{2}{|c|}{3.249 to $77.617^{\circ}$} \\
\hline Index ranges & \multicolumn{2}{|c|}{$-8<=\mathrm{h}<=9,-7<=\mathrm{k}<=8,-69<=1<=66$} \\
\hline Reflections collected & \multicolumn{2}{|l|}{24352} \\
\hline Independent reflections & \multicolumn{2}{|c|}{$2924[\mathrm{R}(\mathrm{int})=0.0278]$} \\
\hline Completeness to theta $=67.684^{\circ}$ & \multicolumn{2}{|c|}{$100.0 \%$} \\
\hline Absorption correction & \multicolumn{2}{|c|}{ Semi-empirical from equivalents } \\
\hline Max. and min. transmission & \multicolumn{2}{|c|}{1.00000 and 0.69713} \\
\hline Refinement method & \multicolumn{2}{|c|}{ Full-matrix least-squares on $\mathrm{F}^{2}$} \\
\hline Data / restraints / parameters & \multicolumn{2}{|l|}{2924 / 0 / 186} \\
\hline Goodness-of-fit on $\mathrm{F}^{2}$ & \multicolumn{2}{|l|}{1.052} \\
\hline Final $R$ indices $[\mathrm{I}>2 \operatorname{sigma}(\mathrm{I})]$ & \multicolumn{2}{|c|}{$\mathrm{R} 1=0.0387, \mathrm{wR} 2=0.1006$} \\
\hline $\mathrm{R}$ indices (all data) & \multicolumn{2}{|c|}{$\mathrm{R} 1=0.0397, \mathrm{wR} 2=0.1015$} \\
\hline Extinction coefficient & \multicolumn{2}{|l|}{$0.00058(11)$} \\
\hline Largest diff. peak and hole & \multicolumn{2}{|c|}{0.294 and -0.222 e. $\AA^{-3}$} \\
\hline
\end{tabular}


<smiles>O=C1CCCC=C1I</smiles><smiles>Nc1ccc(-c2ccccc2)cc1</smiles>

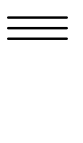<smiles>CC1C(C)C(C)C(C(C)C2C(C)C(C)C(C3C(C)C(C)C(C)C(C)C3C)C(C)C2C)C(C)C1C</smiles>

Figure S1: Crystallographic Data for 3i. Crystal structure with ellipsoid contour at 50\% probability level. Single crystal of $3 \mathbf{i}\left[\mathbf{C}_{18} \mathbf{H}_{17} \mathbf{N O}\right]$ was obtained from the mixed solvents of DCM and n-hexane. The X-ray crystallographic data were collected using a SMART APEX II Diffractometer. CCDC 2062654 contains the supplementary crystallographic data which can be obtained free of charge from The Cambridge Crystallographic Data Center via www.ccdc.cam.ac.uk/data_request/cif.

\section{Extra optimization for the reaction conditions.}

\section{a. $\alpha$-Enaminone formation reaction (Table $\mathbf{S 1})^{\mathrm{a}}$}

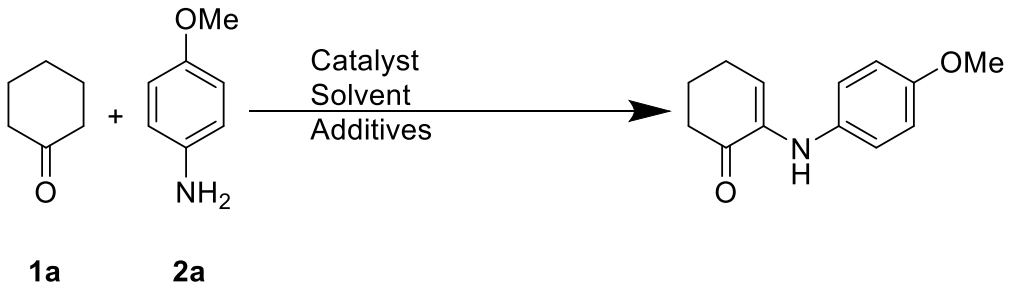

\begin{tabular}{|c|c|c|c|c|c|c|}
\hline Entry & Cat. (mol\%) & $1 \mathbf{a}(\mathrm{mmol})$ & 2a (mmol) & Solvent (mL) & Additives & Yield $(\%)^{b}$ \\
\hline 1 & TfOH (2) & 4.0 & 0.2 & Toluene (1.0) & $\mathrm{O}_{2}$ Bubbled & 9 \\
\hline 2 & TfOH (5) & 4.0 & 0.2 & Toluene (1.0) & $\mathrm{O}_{2}$ Bubbled & 12 \\
\hline 3 & TfOH (30) & 4.0 & 0.2 & Toluene (1.0) & $\mathrm{O}_{2}$ Bubbled & 17 \\
\hline 4 & TfOH (10) & 0.1 & 0.2 & Toluene (1.0) & $\mathrm{O}_{2}$ Bubbled & 5 \\
\hline 5 & TfOH (10) & 0.2 & 0.2 & Toluene (1.0) & $\mathrm{O}_{2}$ Bubbled & 9 \\
\hline 6 & TfOH (10) & 0.5 & 0.2 & Toluene (1.0) & $\mathrm{O}_{2}$ Bubbled & 16 \\
\hline 7 & TfOH (10) & 1.0 & 0.2 & Toluene (1.0) & $\mathrm{O}_{2}$ Bubbled & 22 \\
\hline 8 & TfOH (10) & 2.0 & 0.2 & Toluene (1.0) & $\mathrm{O}_{2}$ Bubbled & 28 \\
\hline 9 & TfOH (10) & Neat & 0.2 & Toluene (1.0) & $\mathrm{O}_{2}$ Bubbled & 19 \\
\hline 10 & TfOH (10) & 4.0 & 0.2 & Toluene (1.0) & $\mathrm{Ar}$ & Trace \\
\hline
\end{tabular}

${ }^{a}$ Reaction conditions: Unless otherwise noted 1a (4.0mmol), $2 \mathbf{2 a}(0.2 \mathrm{mmol}) .{ }^{b}$ Estimated by ${ }^{1} \mathrm{H}$ NMR spectroscopy using dibromethane as the internal standard. 


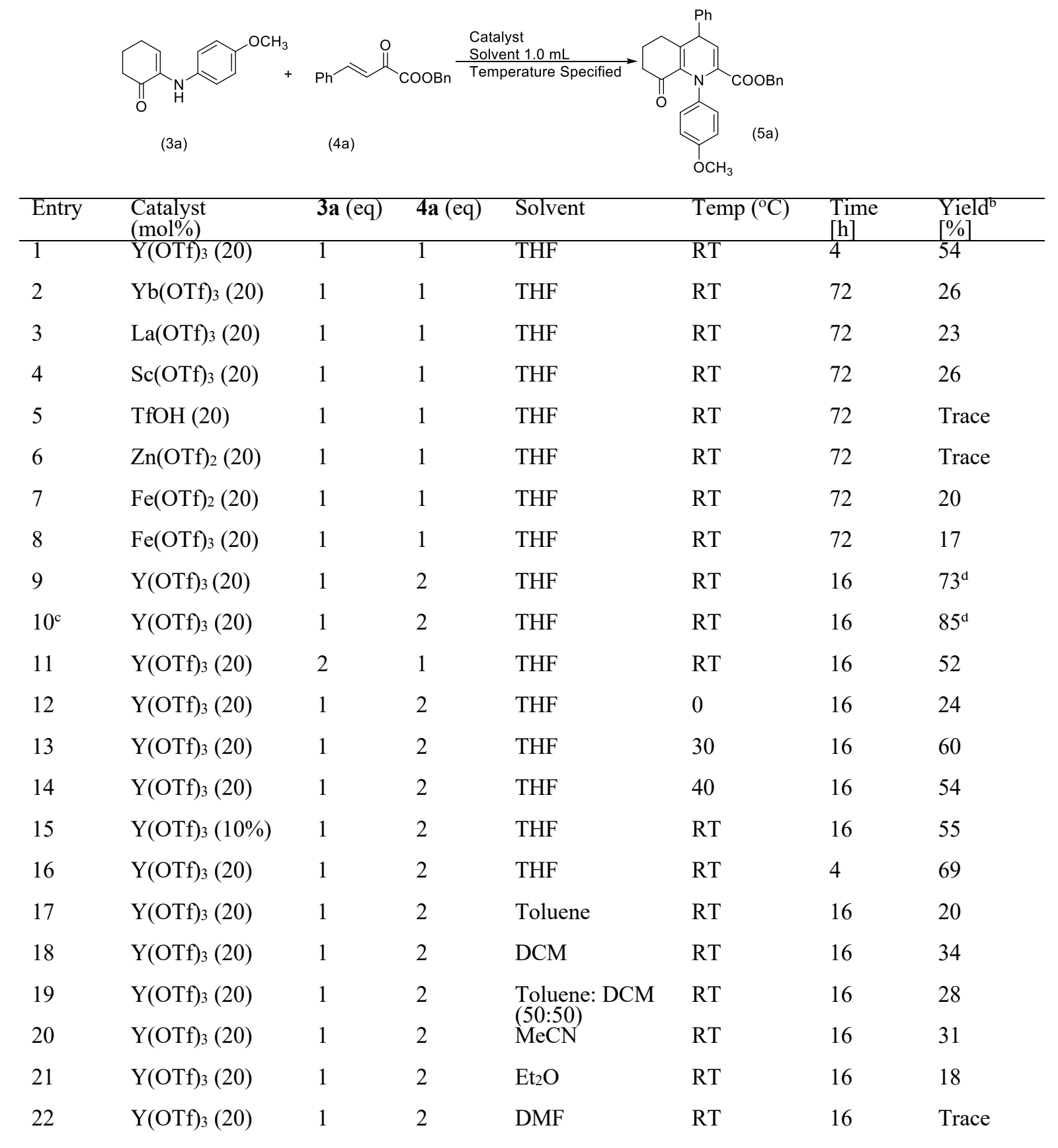

${ }^{a}$ Reaction conditions: Unless otherwise noted 3a $(0.1 \mathrm{mmol}), \mathbf{4 a}(0.1 \mathrm{mmol}) .{ }^{b}$ Estimated by ${ }^{1} \mathrm{H}$ NMR spectroscopy using dibromethane as the internal standard. ${ }^{\mathrm{C}}$ Reaction was performed under argon. ${ }^{\mathrm{d}}$ Isolated yield. 
c. Catalyst loading and additive experiments of [3+3] Cycloaddition Reaction of $\alpha-$ Enaminones and Enones (Table S3) ${ }^{\mathrm{a}}$

\begin{tabular}{|c|c|c|c|c|c|c|}
\hline Entry & Cat. (mol\%) & 3a (mmol) & $\mathbf{4 a}(\mathrm{mmol})$ & Solvent (mL) & Additives & $\begin{array}{l}\text { Yield } \\
(\%)^{b}\end{array}$ \\
\hline 1 & $\mathrm{Y}(\mathrm{OTf})_{3}(30)$ & 0.1 & 0.2 & THF (1.0) & - & 69 \\
\hline 2 & $\mathrm{Y}(\mathrm{OTf})_{3}(10)$ & 0.1 & 0.2 & THF (1.0) & - & 55 \\
\hline 3 & $\mathrm{Y}(\mathrm{OTf})_{3}(5)$ & 0.1 & 0.2 & THF (1.0) & - & 40 \\
\hline 4 & $\mathrm{Y}(\mathrm{OTf})_{3}(20)$ & 0.1 & 0.2 & THF (1.0) & $\begin{array}{l}\text { p-Anisidine } \\
(0.05 \mathrm{mmol})\end{array}$ & 71 \\
\hline 5 & $\mathrm{Y}(\mathrm{OTf})_{3}(20)$ & 0.1 & 0.2 & THF (1.0) & $\begin{array}{c}\text { p-Anisidine } \\
(0.1 \mathrm{mmol})\end{array}$ & 70 \\
\hline 6 & $\mathrm{Y}(\mathrm{OTf})_{3}(20)$ & 0.1 & 0.2 & THF (1.0) & 4A MS (10mg) & 62 \\
\hline 7 & $\mathrm{Y}(\mathrm{OTf})_{3}(20)$ & 0.1 & 0.2 & THF (1.0) & 4A MS (10mg) & 57 \\
\hline
\end{tabular}

${ }^{a}$ Reaction conditions: Unless otherwise noted 3a $(0.1 \mathrm{mmol}), \mathbf{4 a}(0.2 \mathrm{mmol}) .{ }^{b}$ Estimated by ${ }^{1} \mathrm{H}$ NMR spectroscopy using dibromethane as the internal standard.

\section{Mechanism Studies}

a. Control NMR experiments.<smiles>COc1ccc(NC2=CCCCC2=O)cc1</smiles><smiles>COc1ccc(NC2=CCCCC2)cc1</smiles>
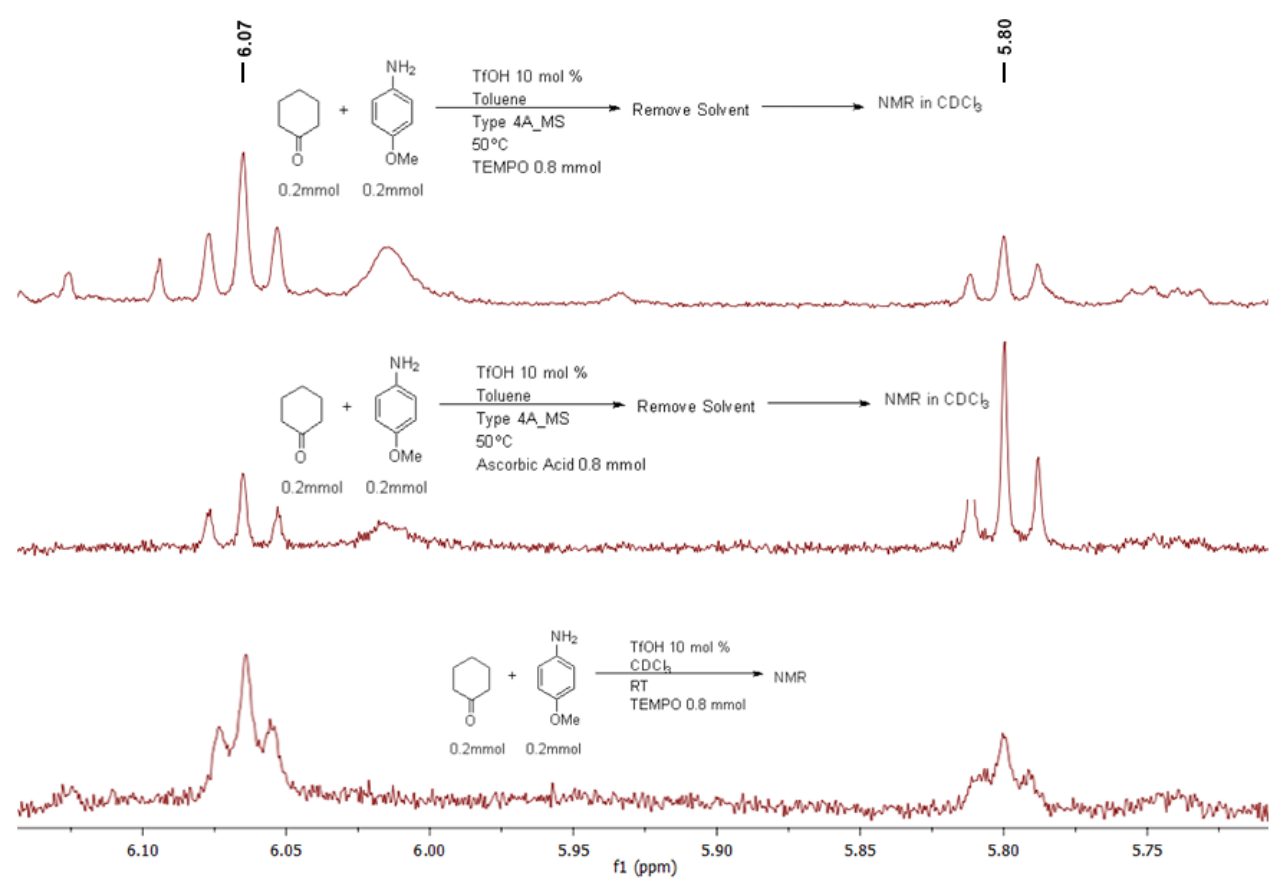

Figure S2: Free-radical scavenger study and the observation of enamine. 


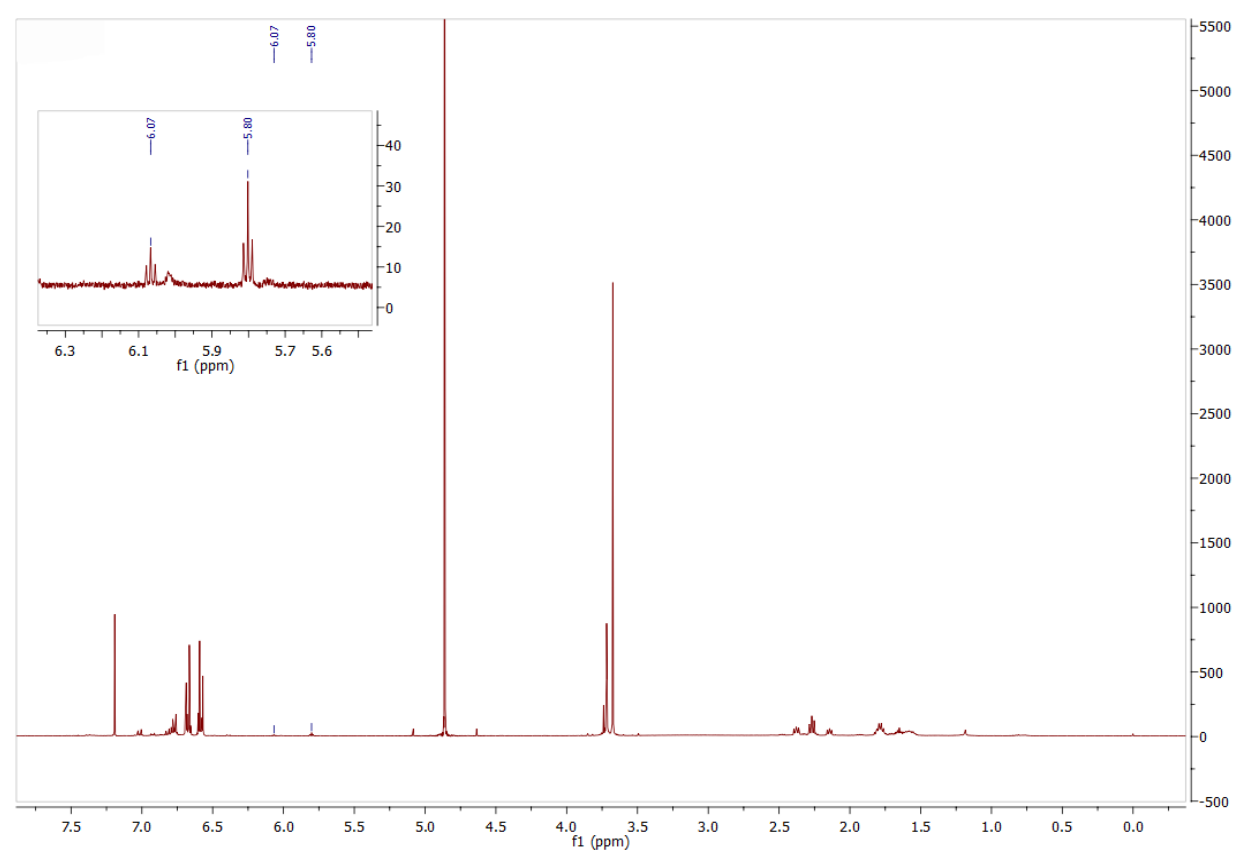

Figure S3: Full crude NMR of (b) in Figure S2

b. 2D NMR experiments.

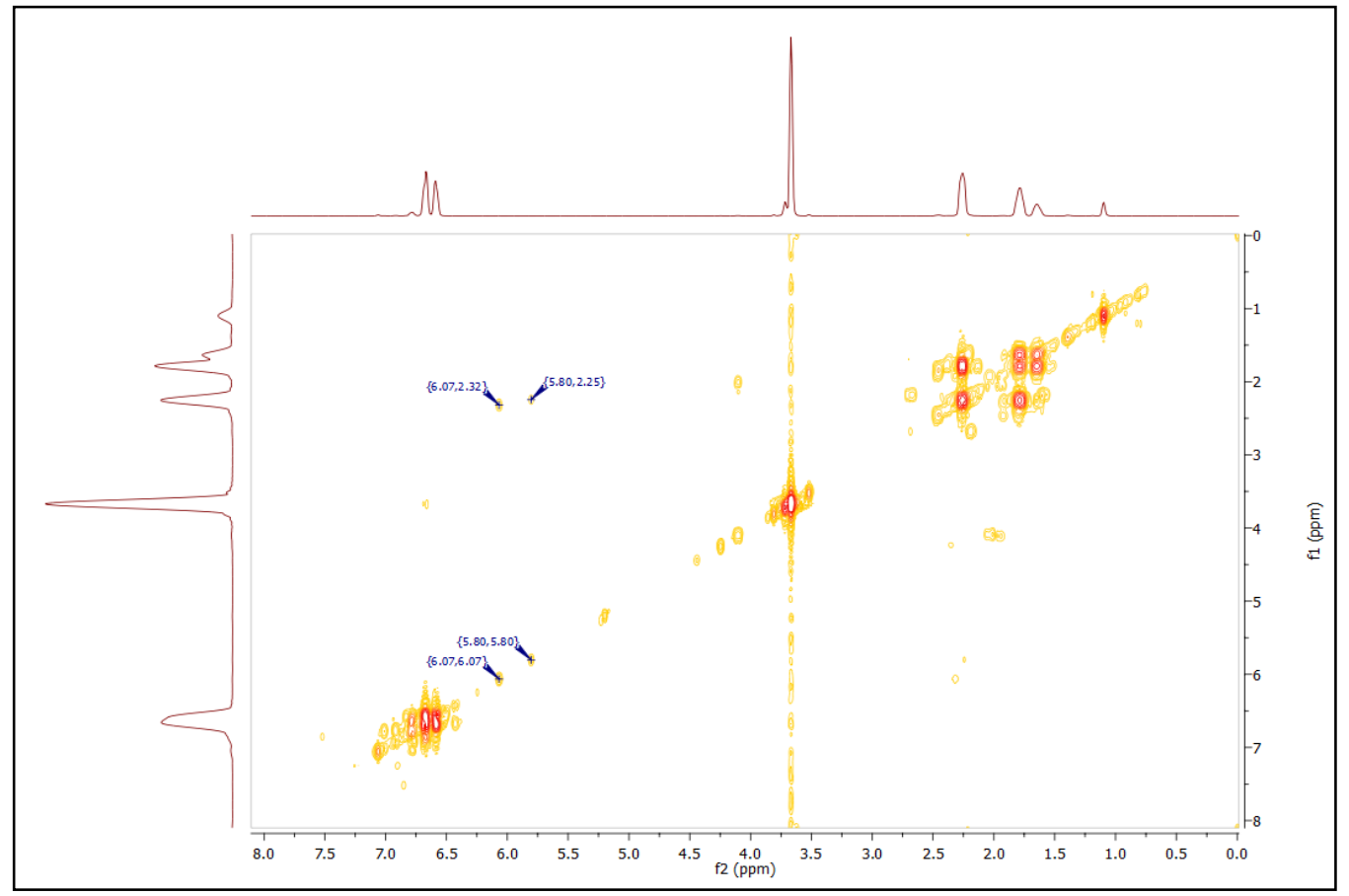

Figure S4: 2D COSY NMR of the control reaction 


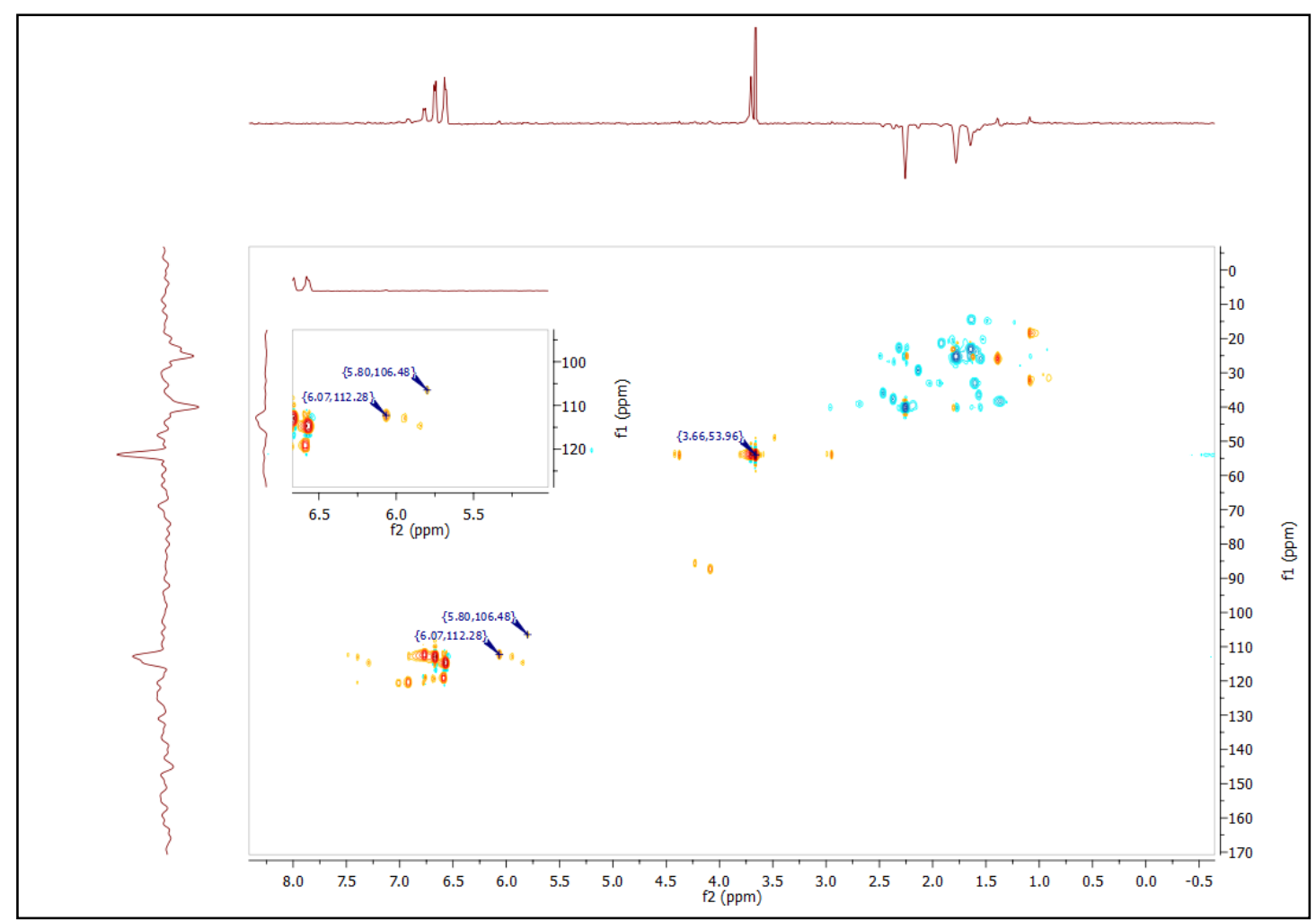

Figure S5: 2D HSQC NMR of the control reaction

\section{Copies of NMR spectra}<smiles>COc1ccc(NC2=CCCCC2=O)cc1</smiles> 

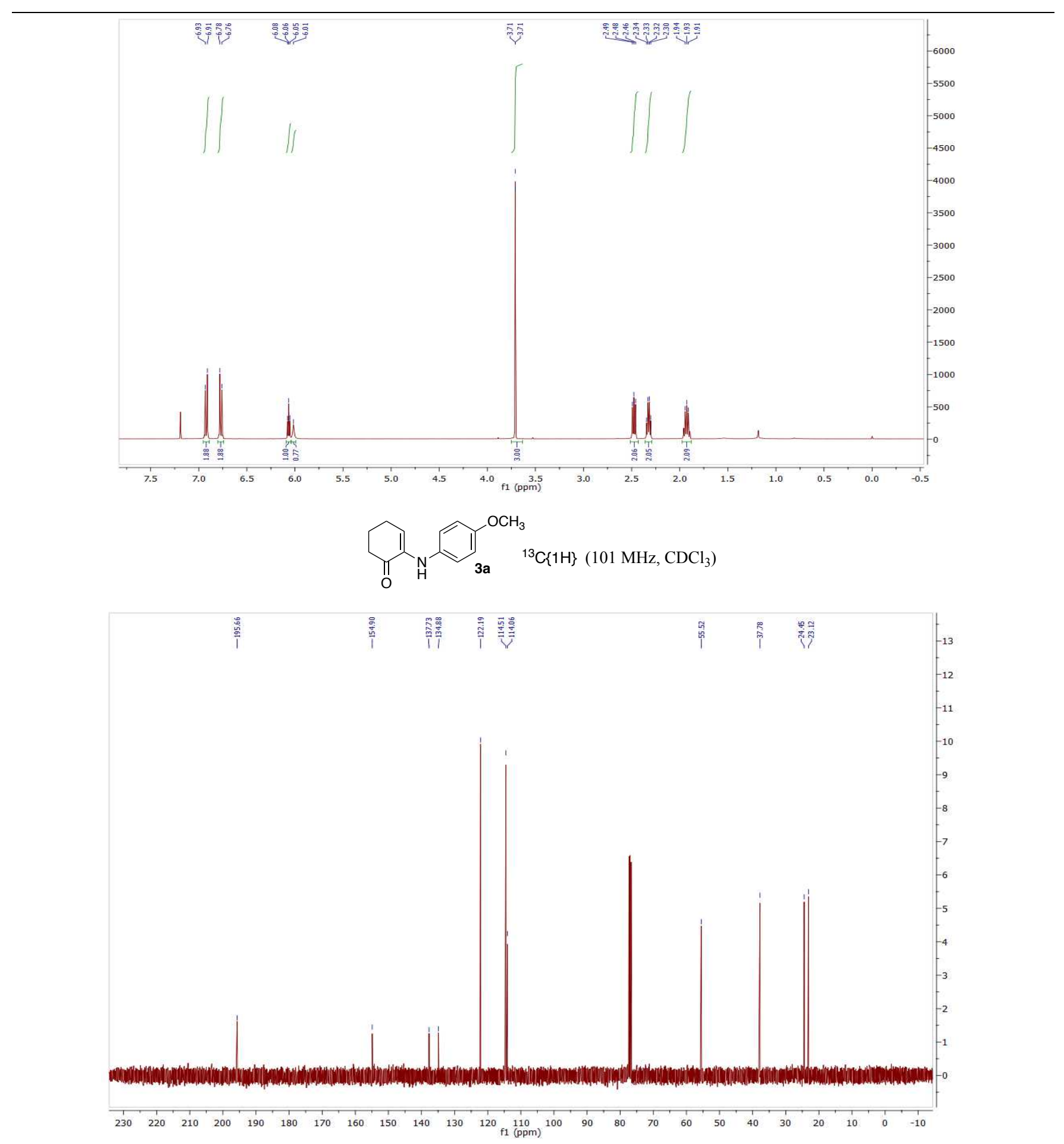

$\prod_{\mathbf{3}} 400 \mathrm{MHz}, \mathrm{CDCl}_{3}$ 

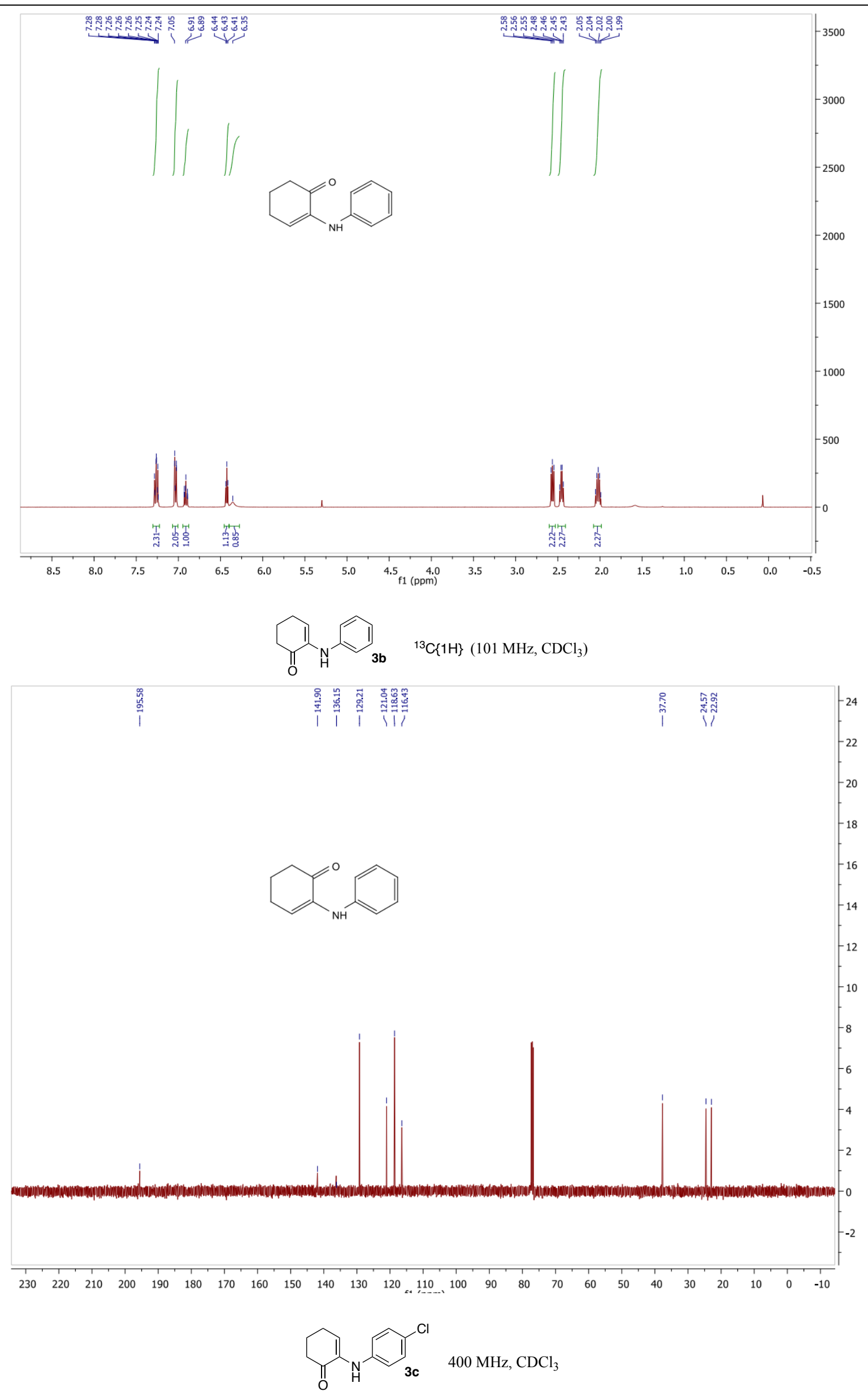


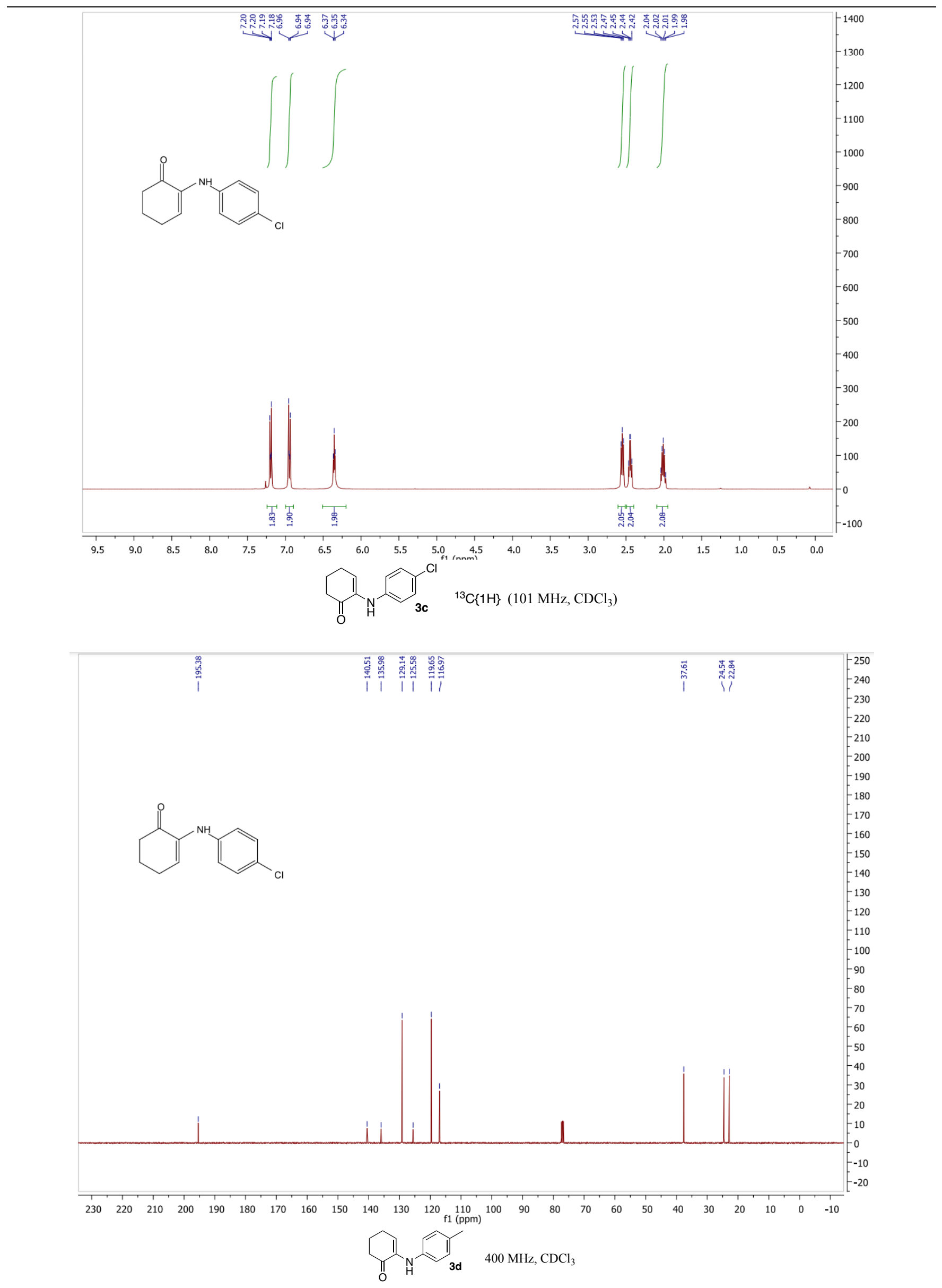

10 


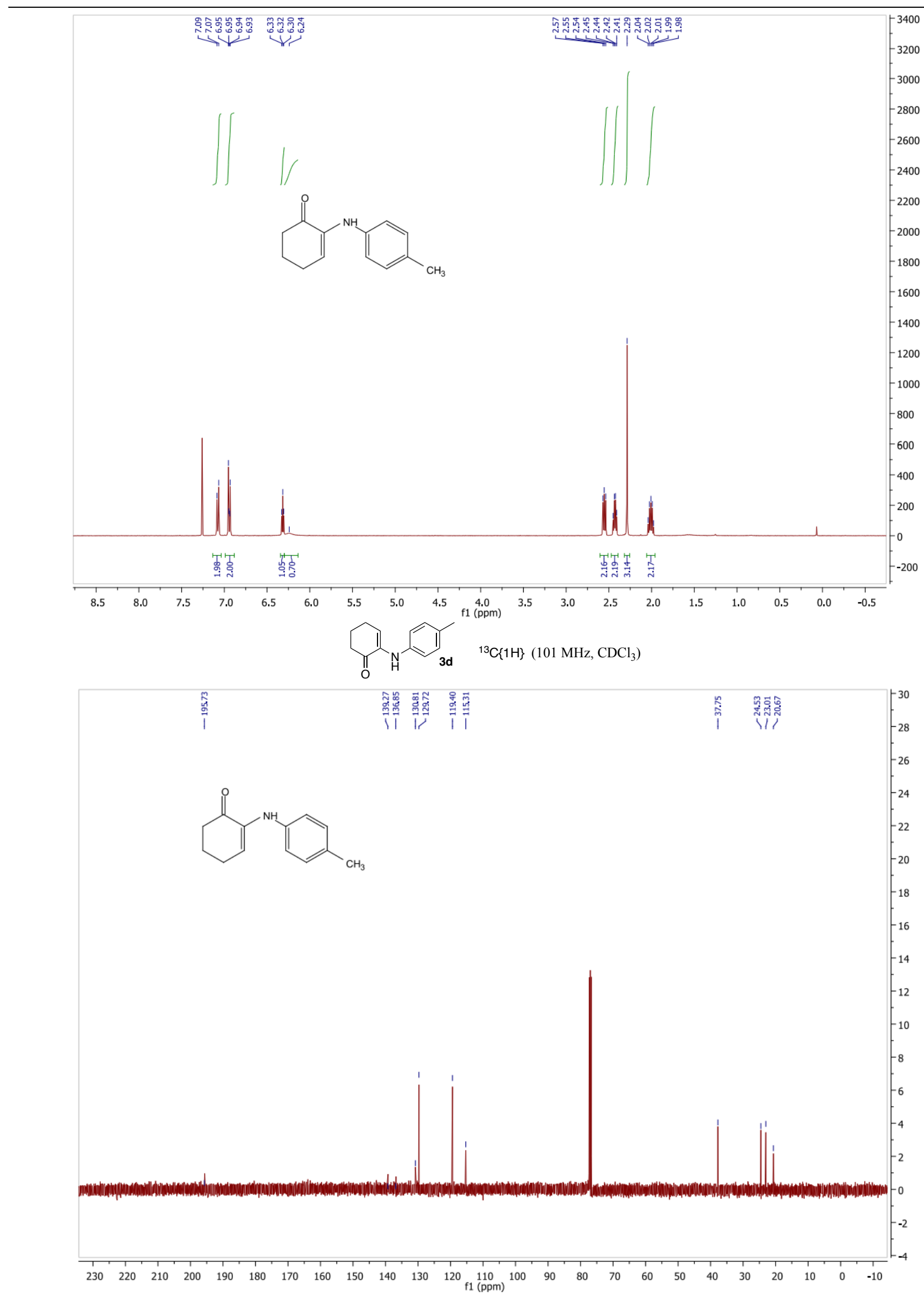

11 


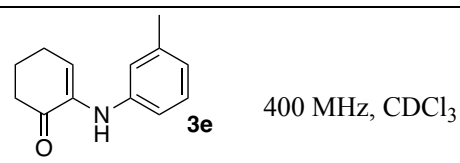

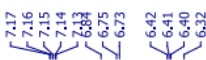

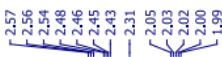
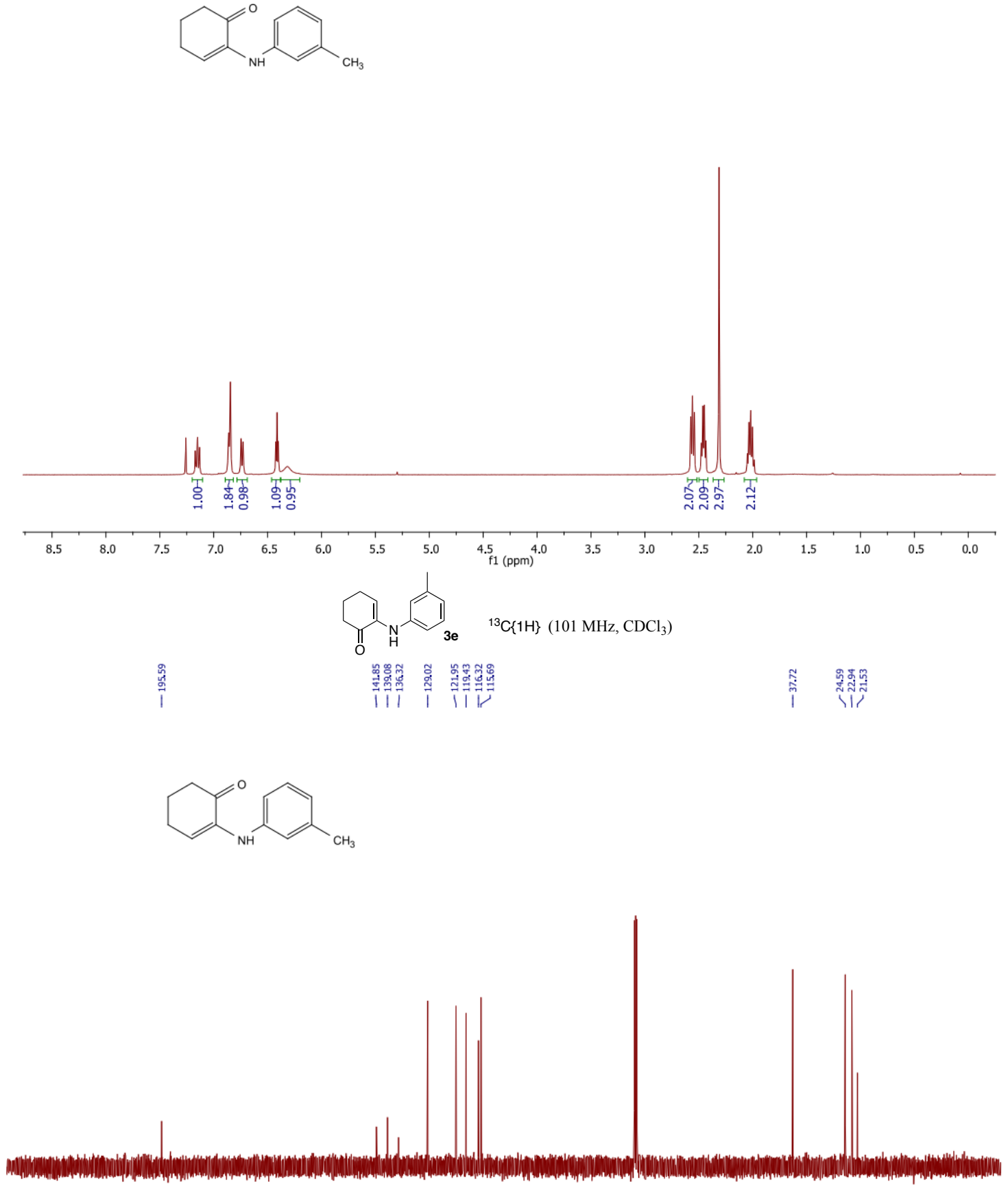

$\begin{array}{llllllllllllllllllllllllllllllllll}230 & 220 & 210 & 200 & 190 & 180 & 170 & 160 & 150 & 140 & 130 & 120 & 110 & 100 & 90 & 80 & 70 & 60 & 50 & 40 & 30 & 20 & 10 & 0 & -10\end{array}$ 


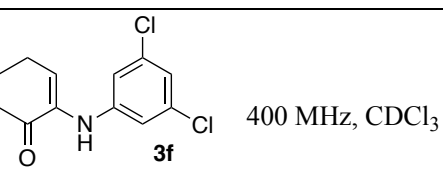

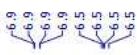

ํำบำกำกำำ
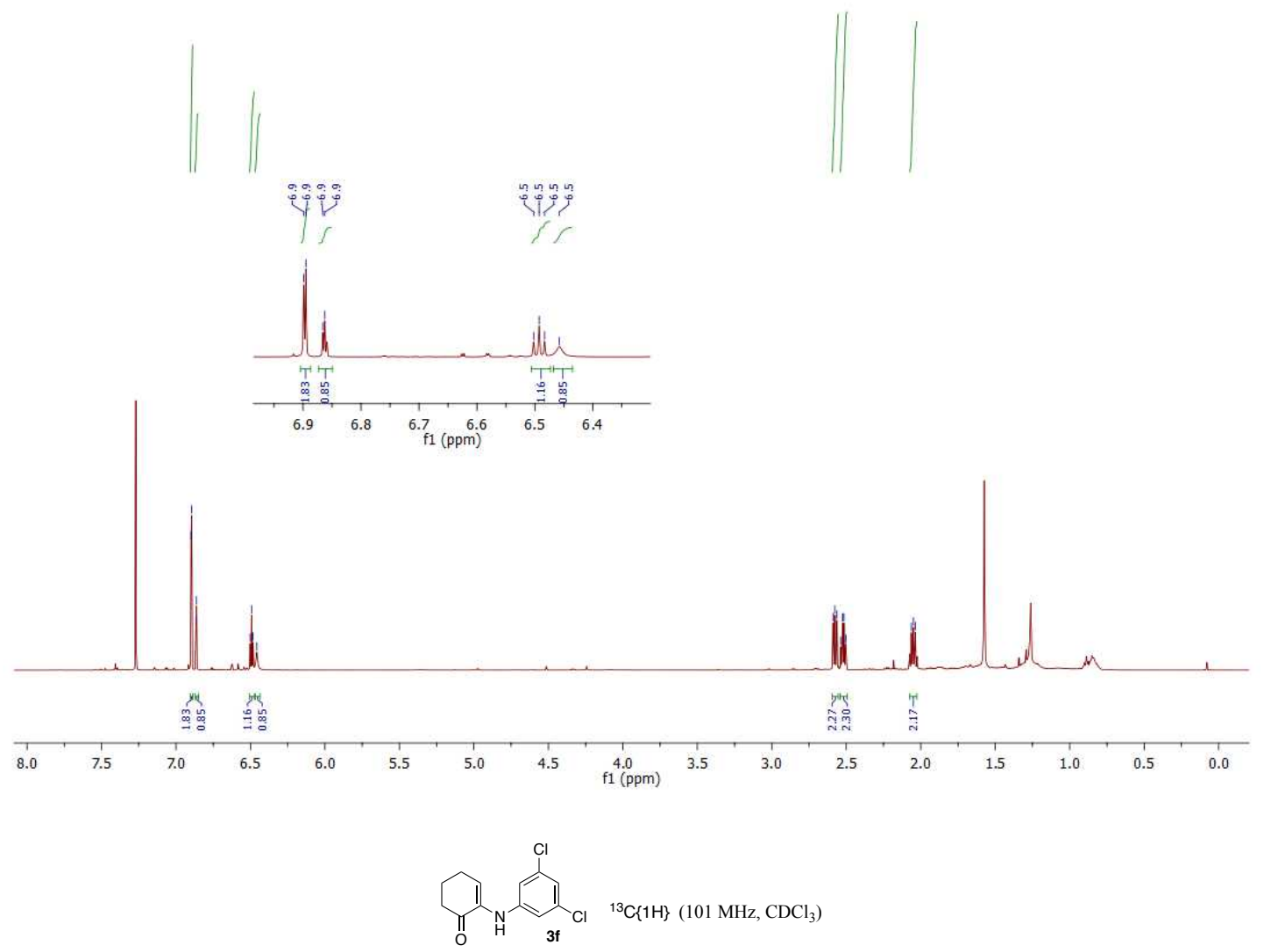

$$
\overrightarrow{\mathrm{i}}
$$

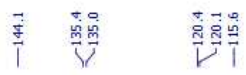

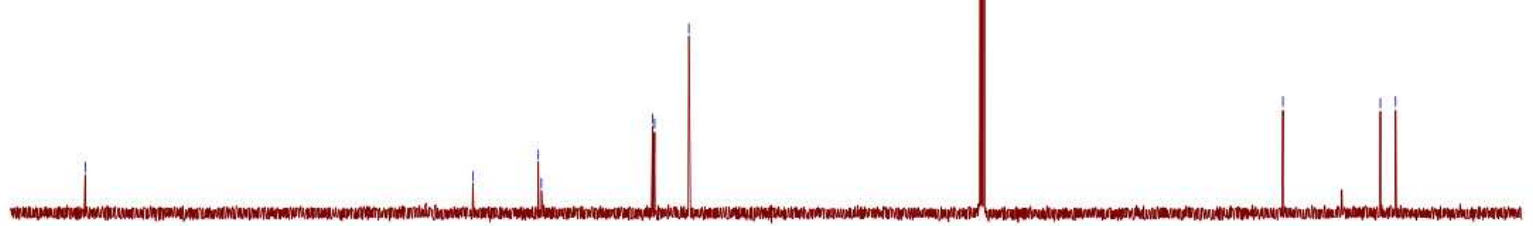

$\begin{array}{llllllllll}200 & 190 & 180 & 170 & 160 & 150 & 140 & 130 & 120 & 110 \\ \mathrm{f} 1(\mathrm{ppm}) & 100\end{array}$ 90 $80 \quad 70$ 
(N) $400 \mathrm{MHz}, \mathrm{CDCl}_{3}$

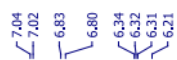

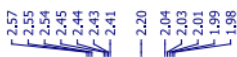

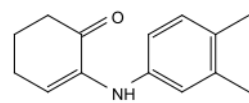

M
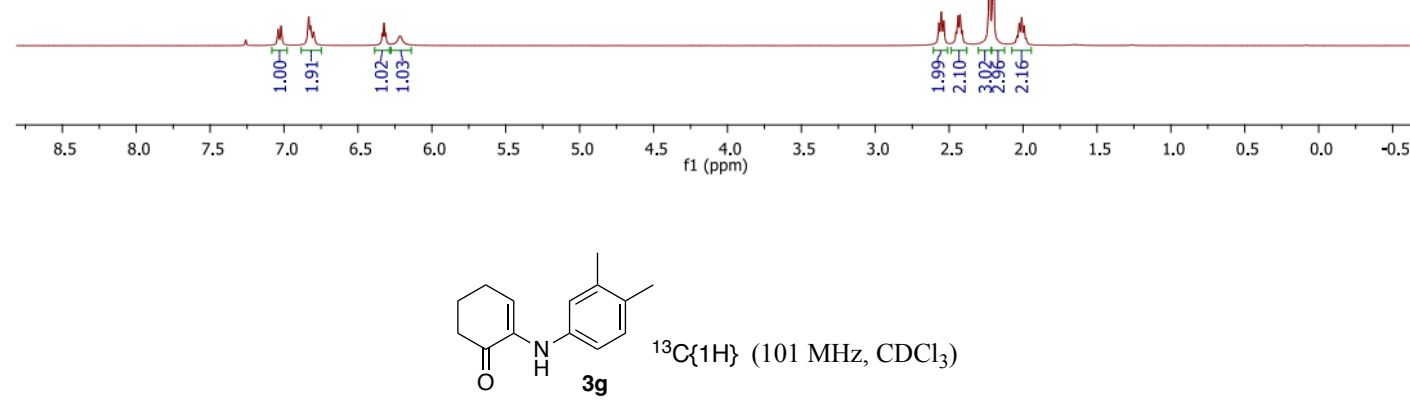

总

望
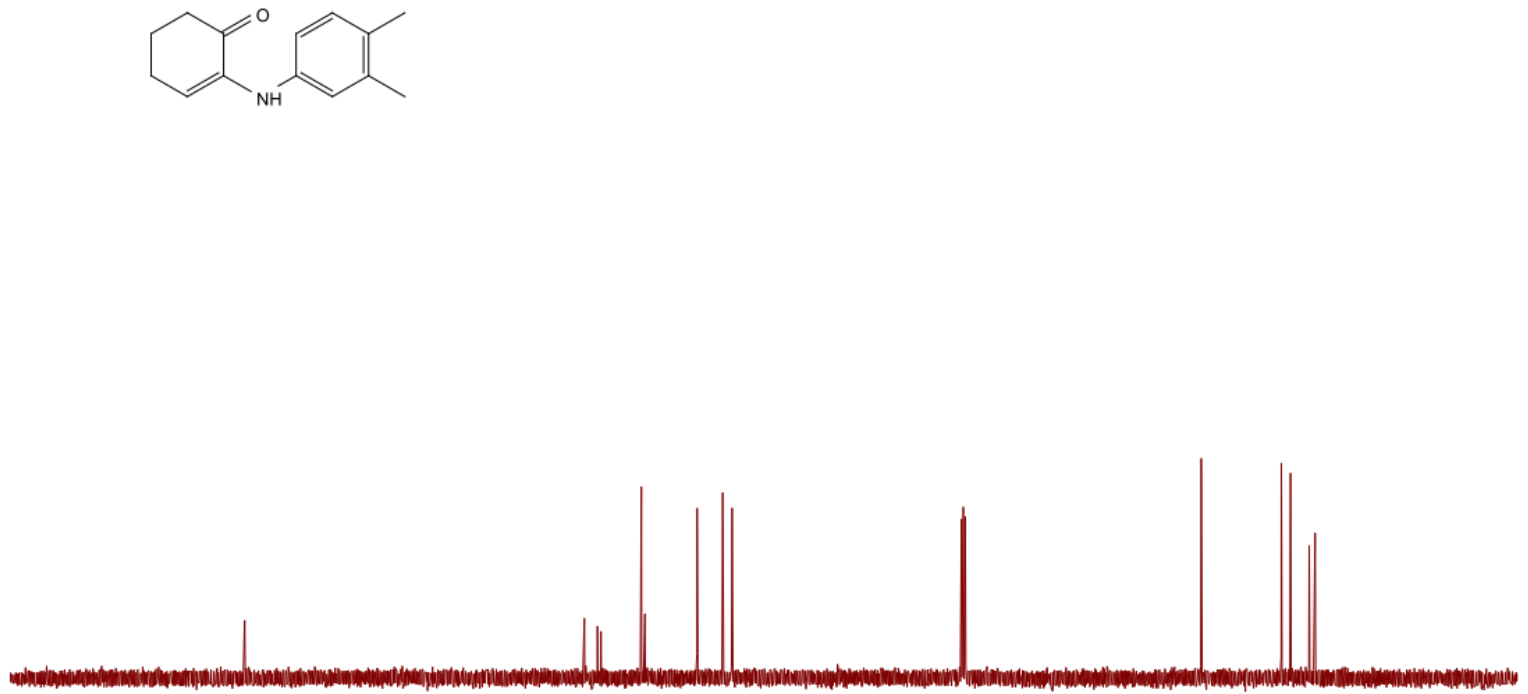

$\begin{array}{lllllllllllllllllllllllllllllllllllllllll}230 & 220 & 210 & 200 & 190 & 180 & 170 & 160 & 150 & 140 & 130 & 120 & 110 & 100 & 90 & 80 & 70 & 60 & 50 & 40 & 30 & 20 & 10 & 0 & -10\end{array}$ 

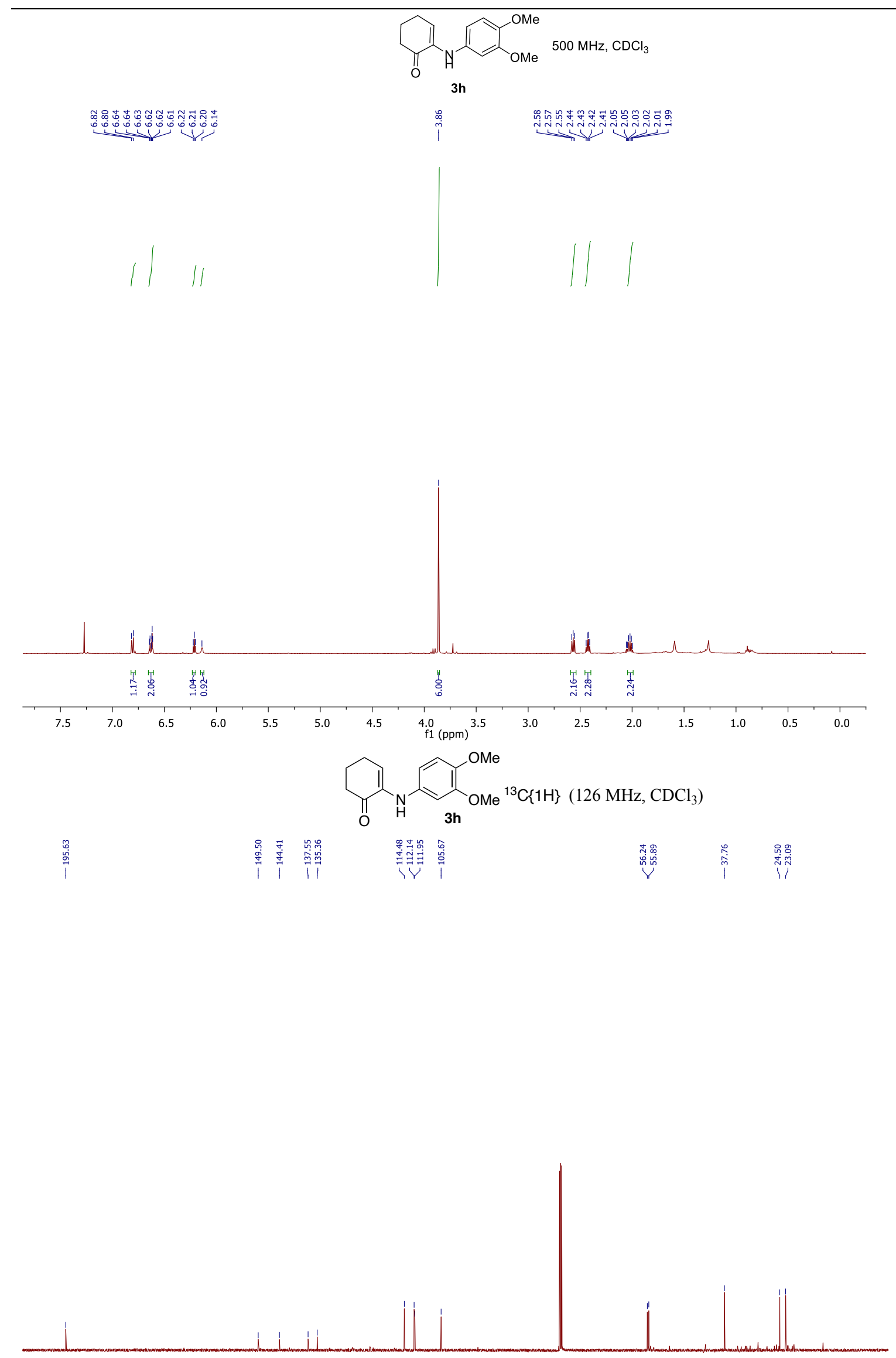

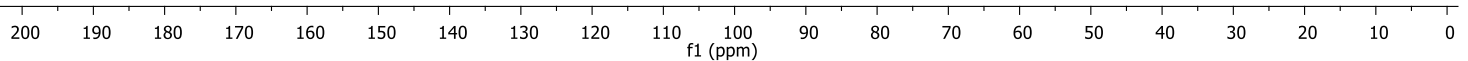



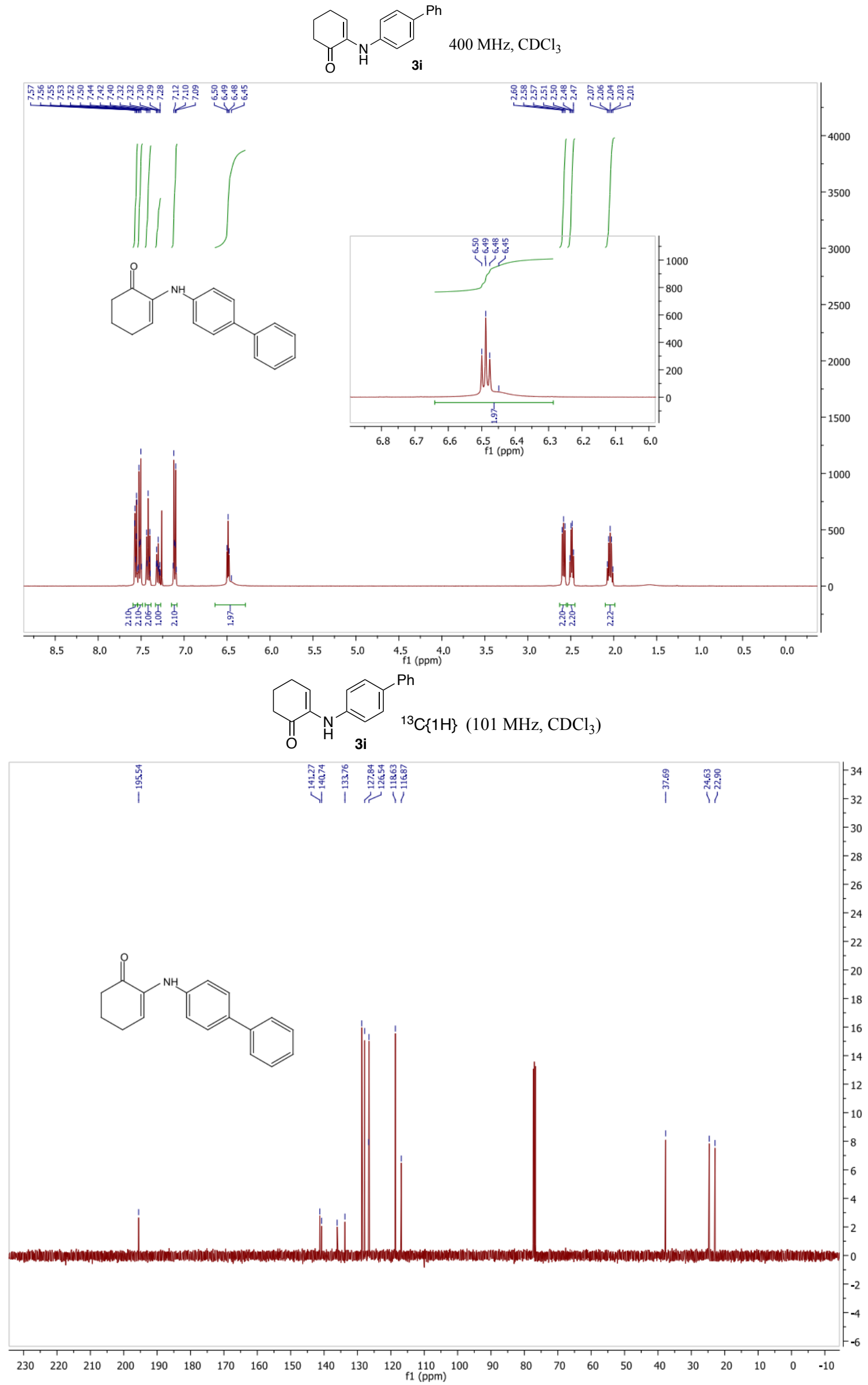

16 


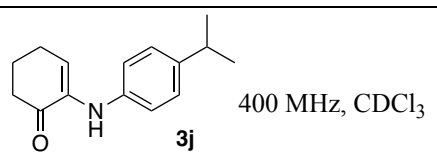

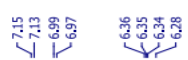

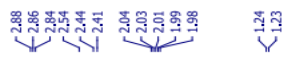

C.

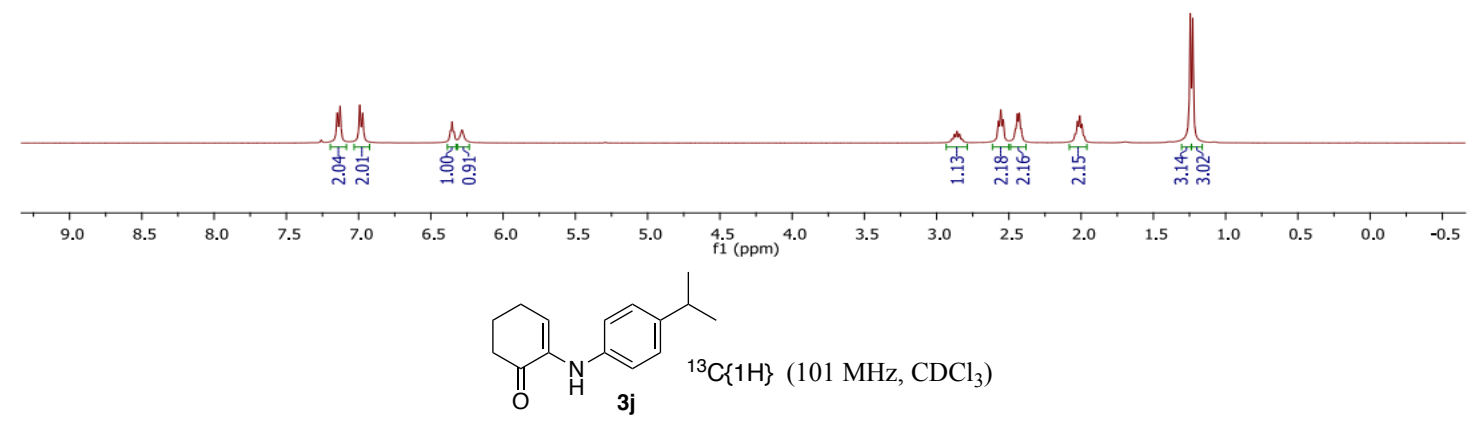

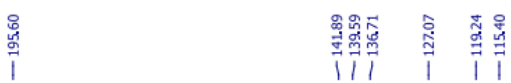

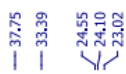
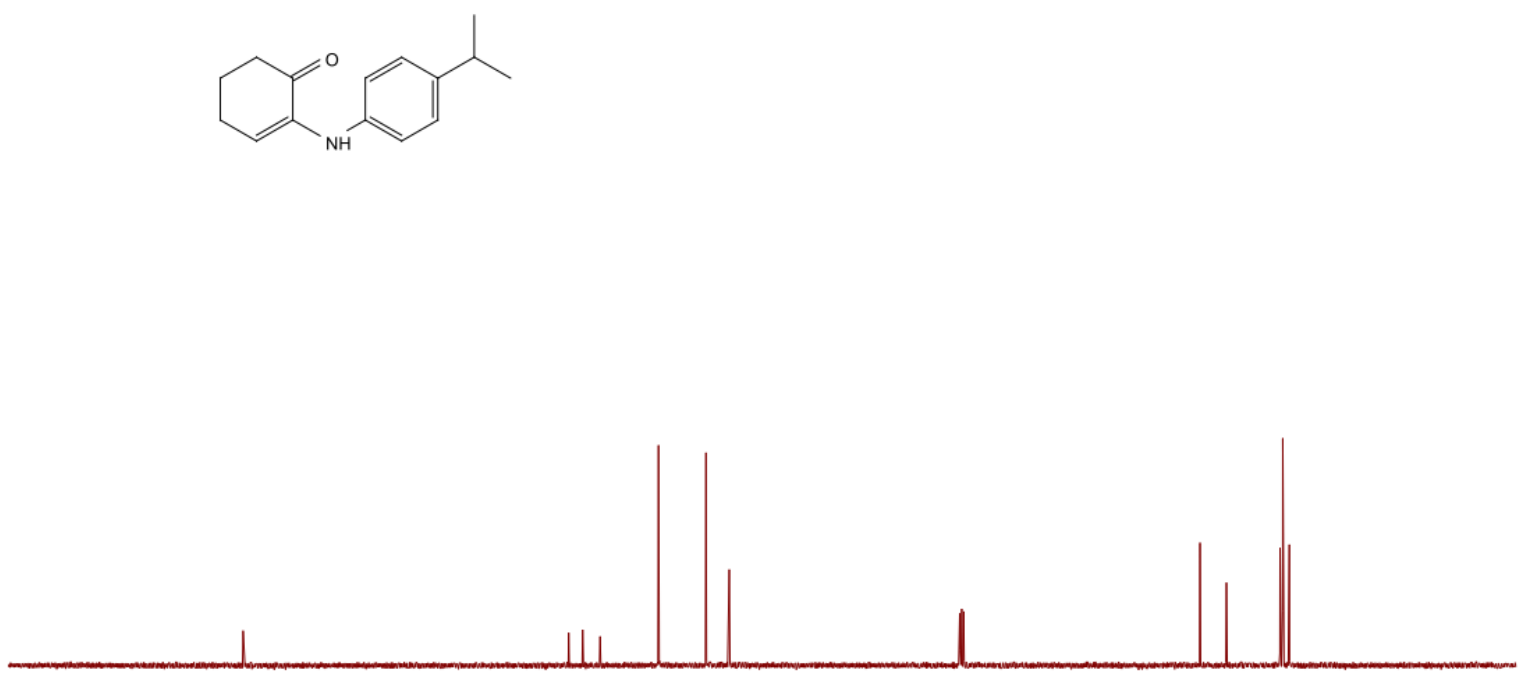

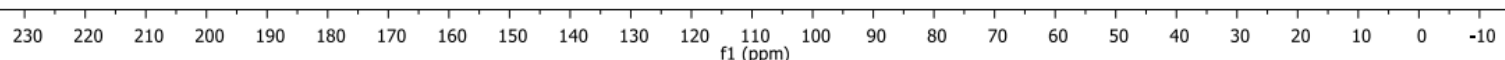



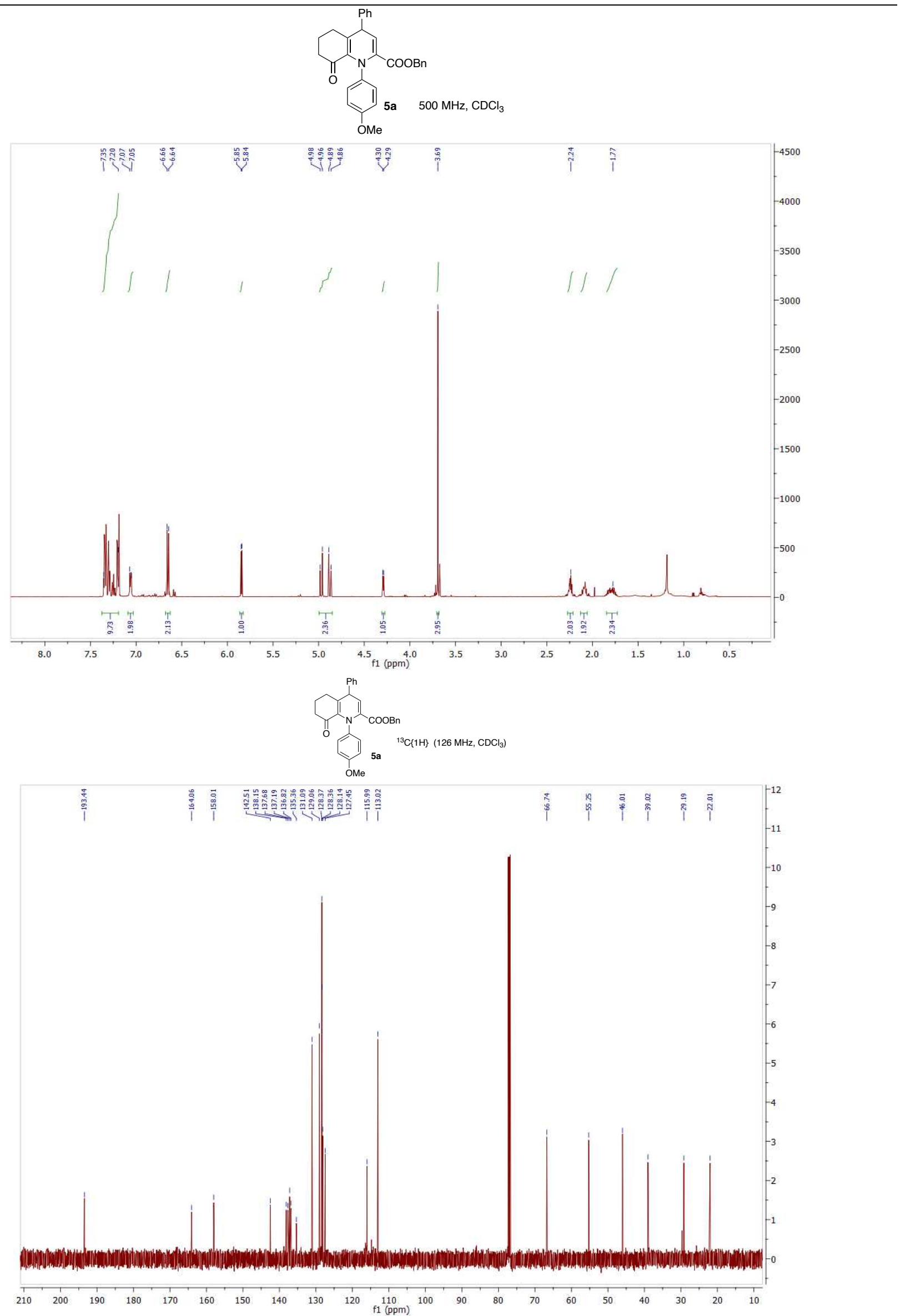

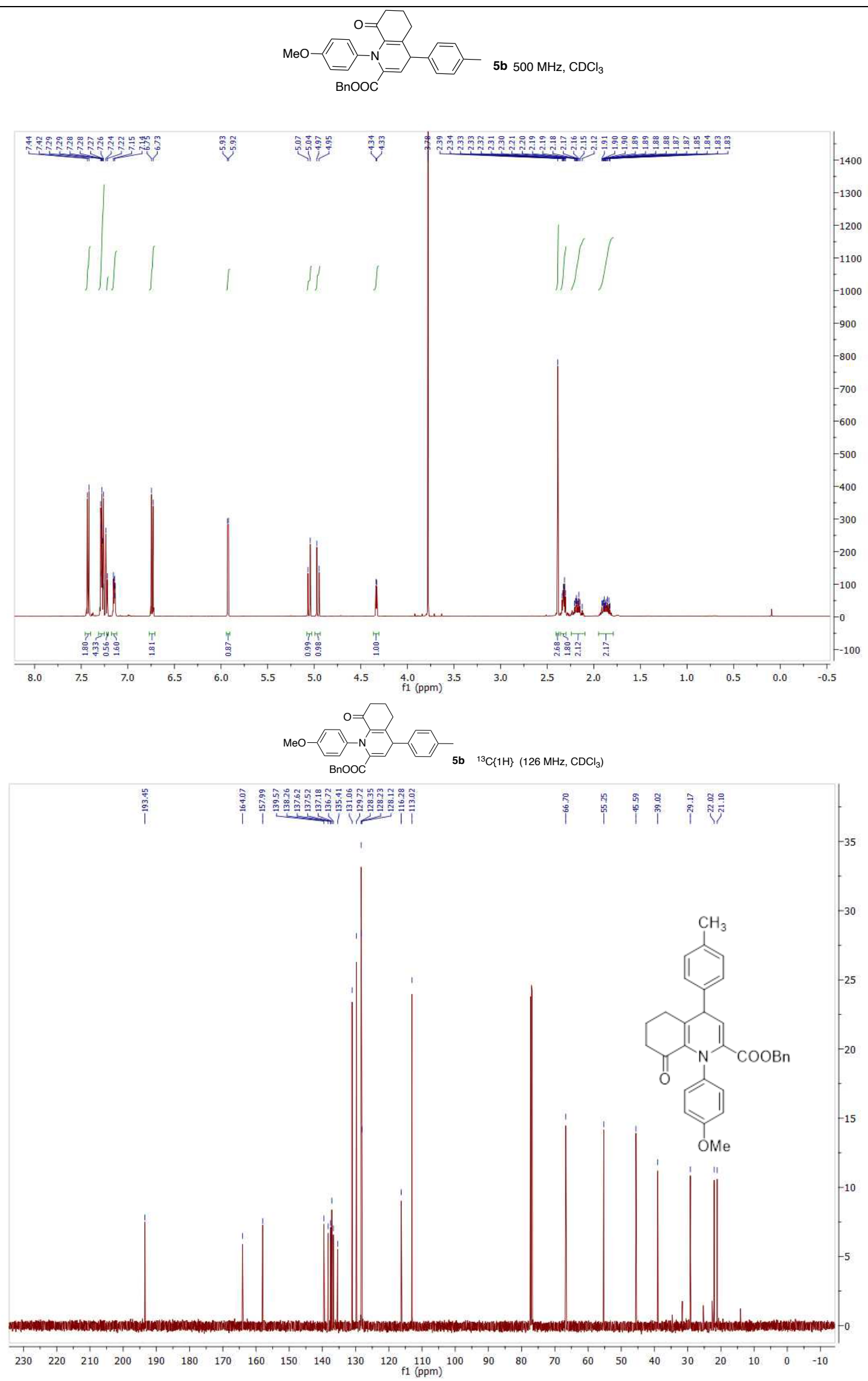

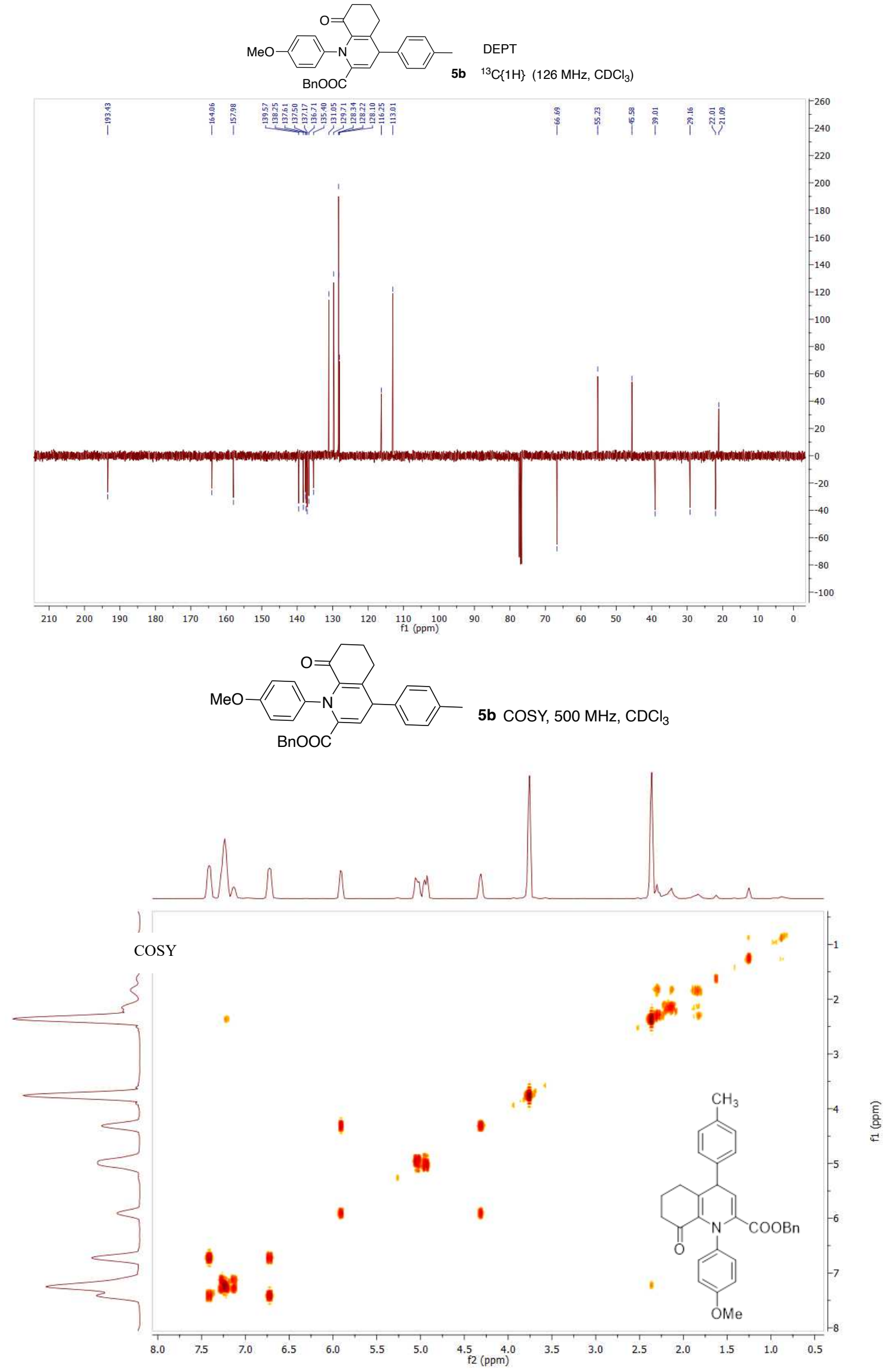

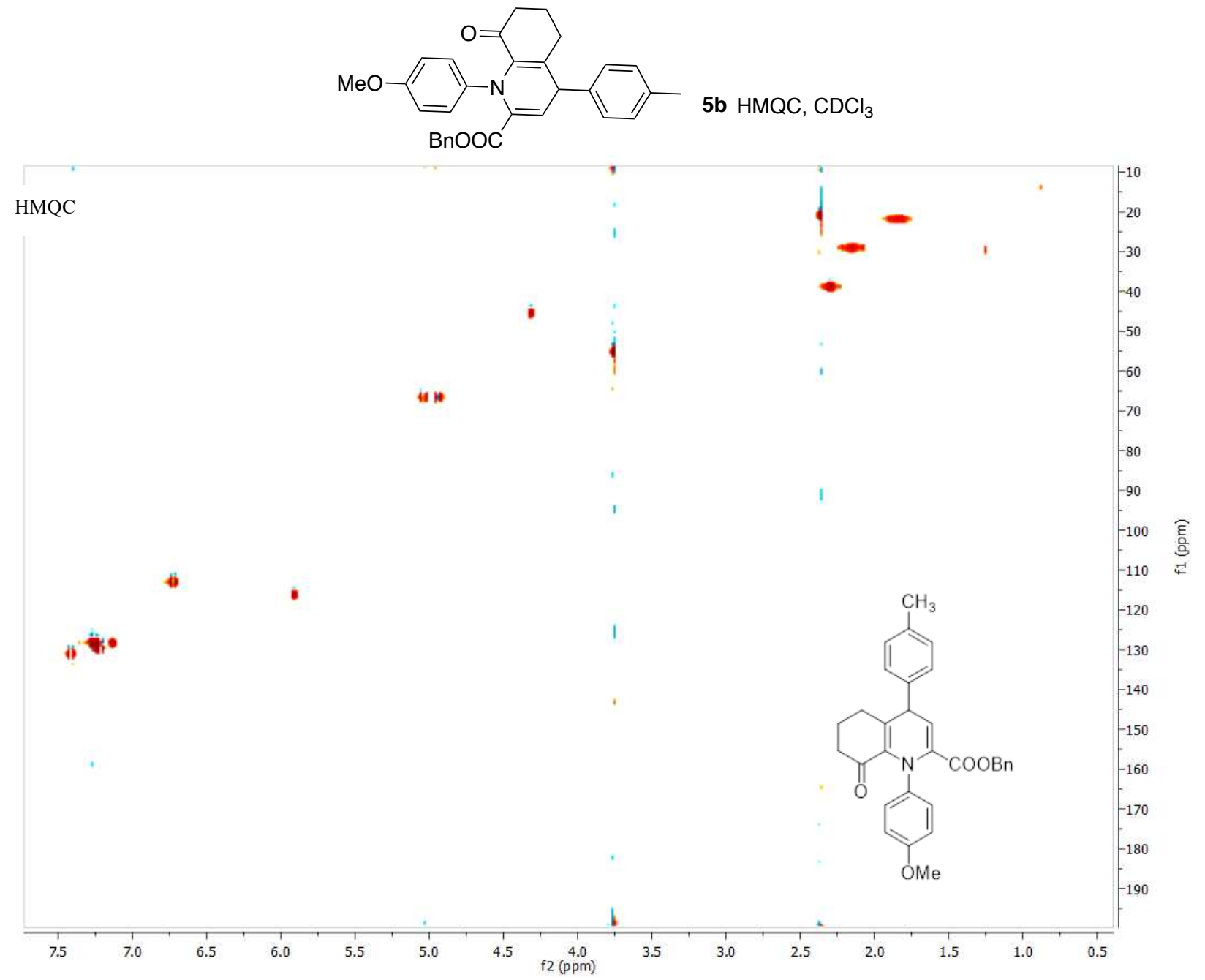

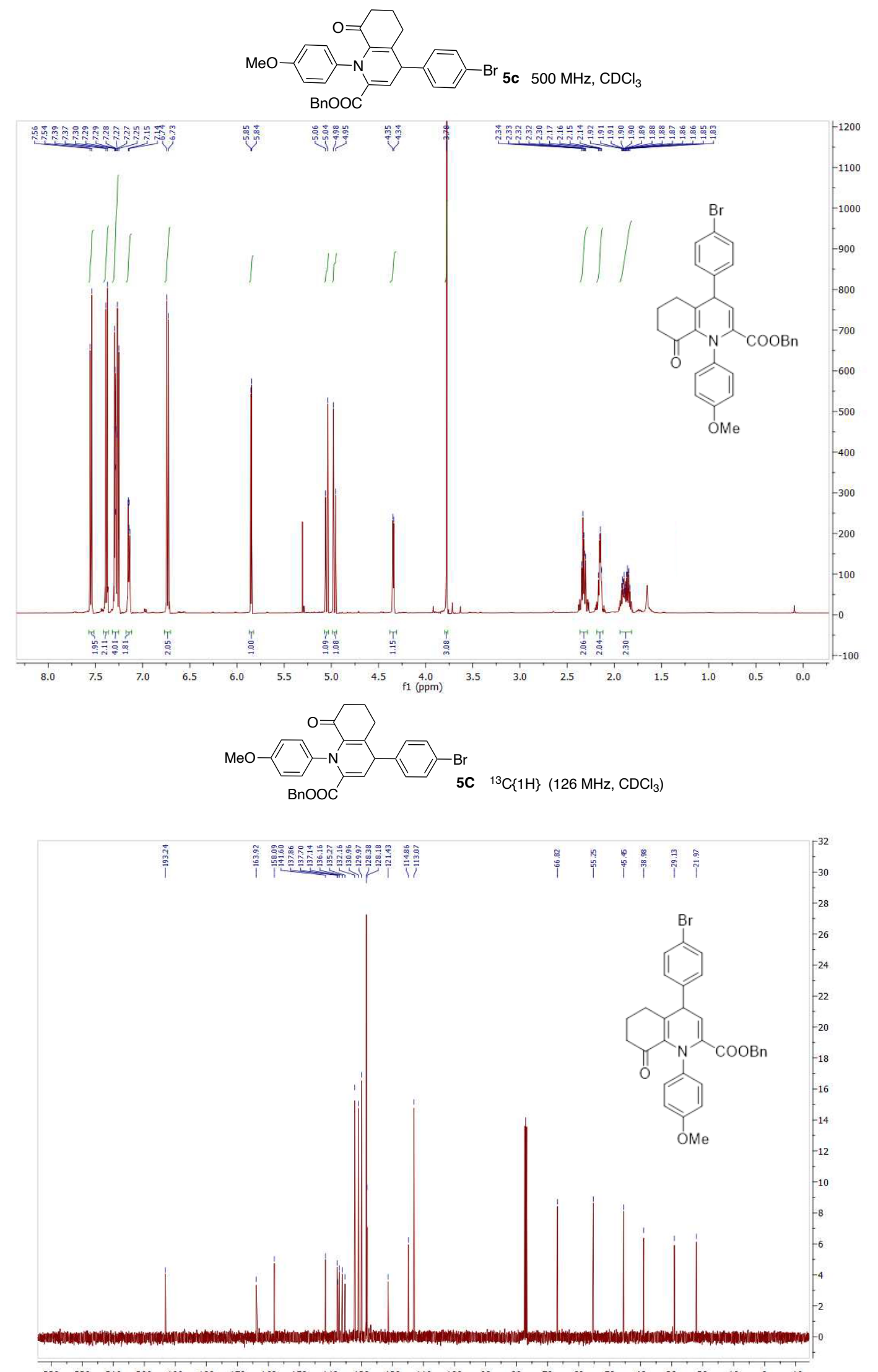

$\begin{array}{lllllllllllllllllllllllllllll}230 & 220 & 210 & 200 & 190 & 180 & 170 & 160 & 150 & 140 & 130 & 120 & 110 & 100 & 90 & 80 & 70 & 60 & 50 & 40 & 30 & 20 & 10 & 0 & -10\end{array}$ 

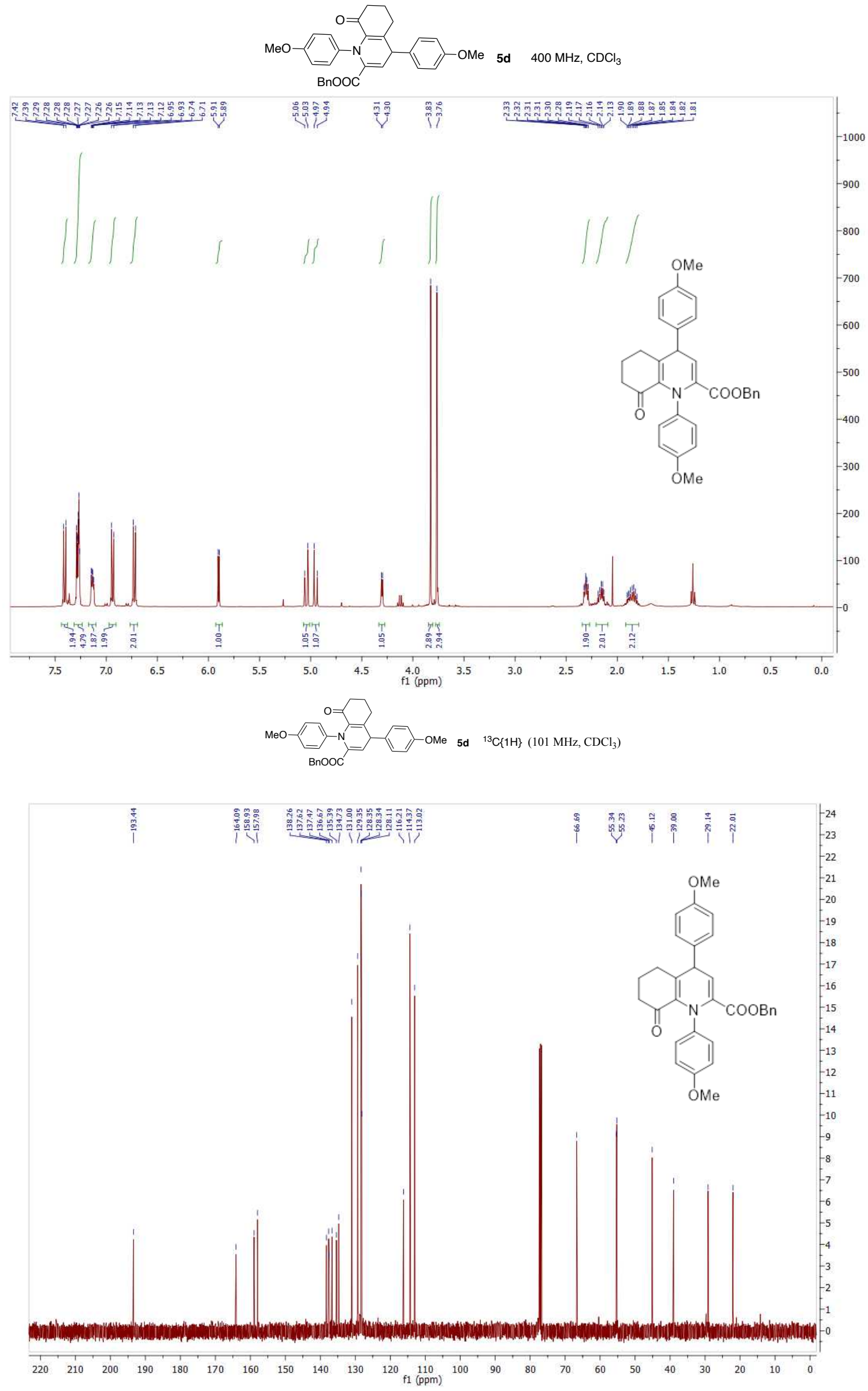


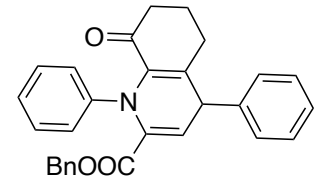

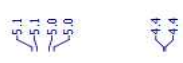

nosis

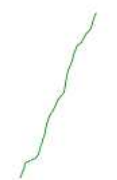

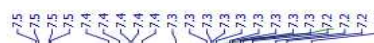
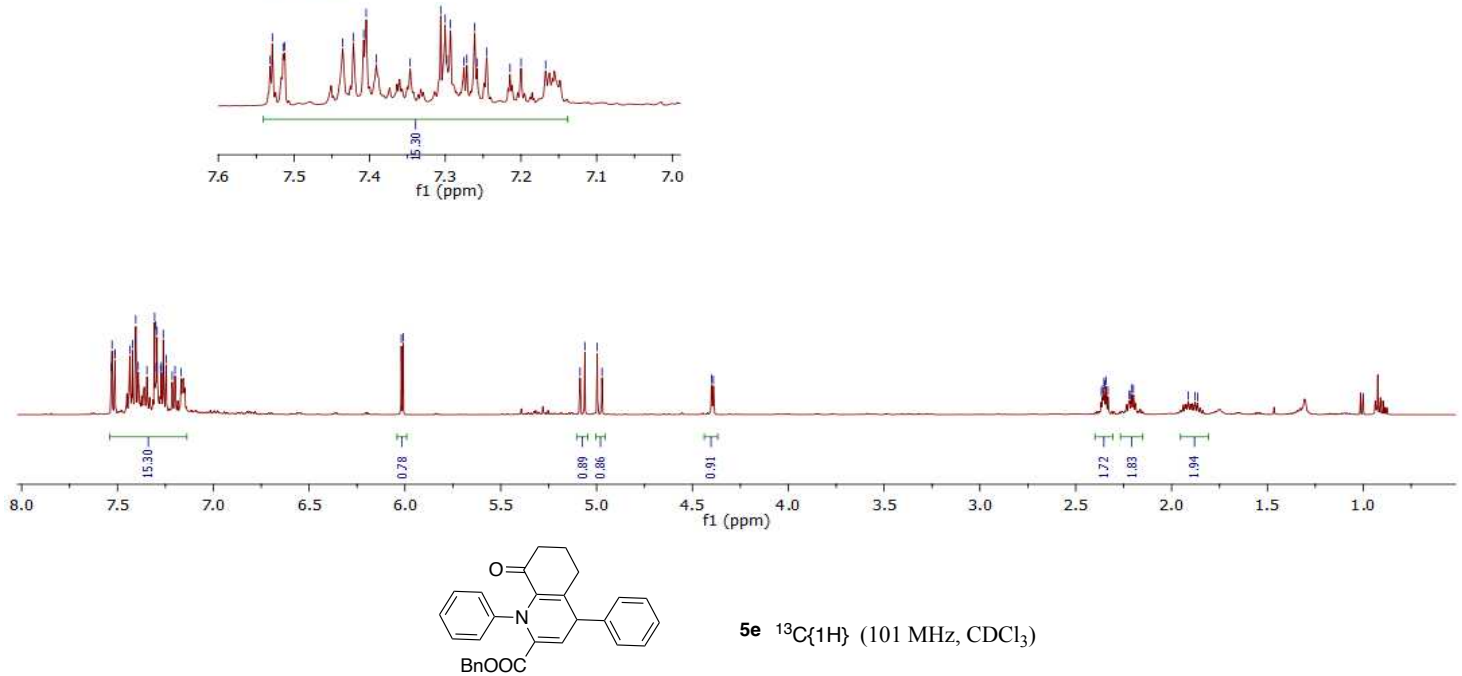

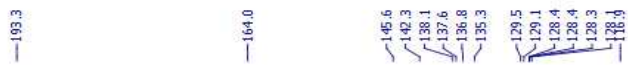

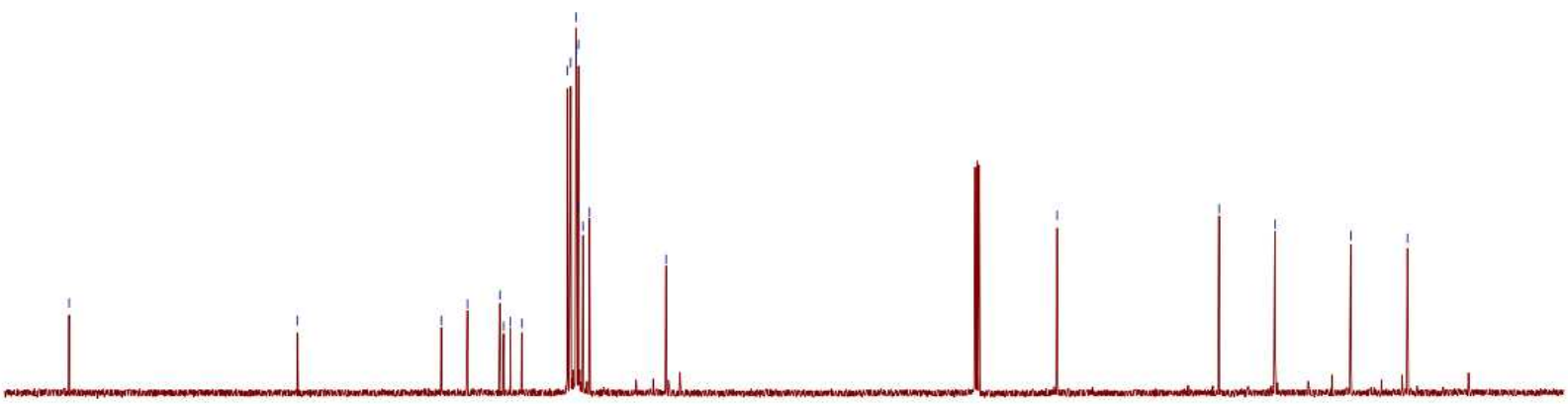

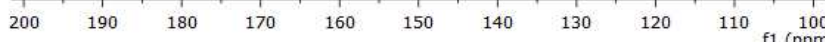

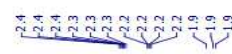

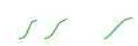



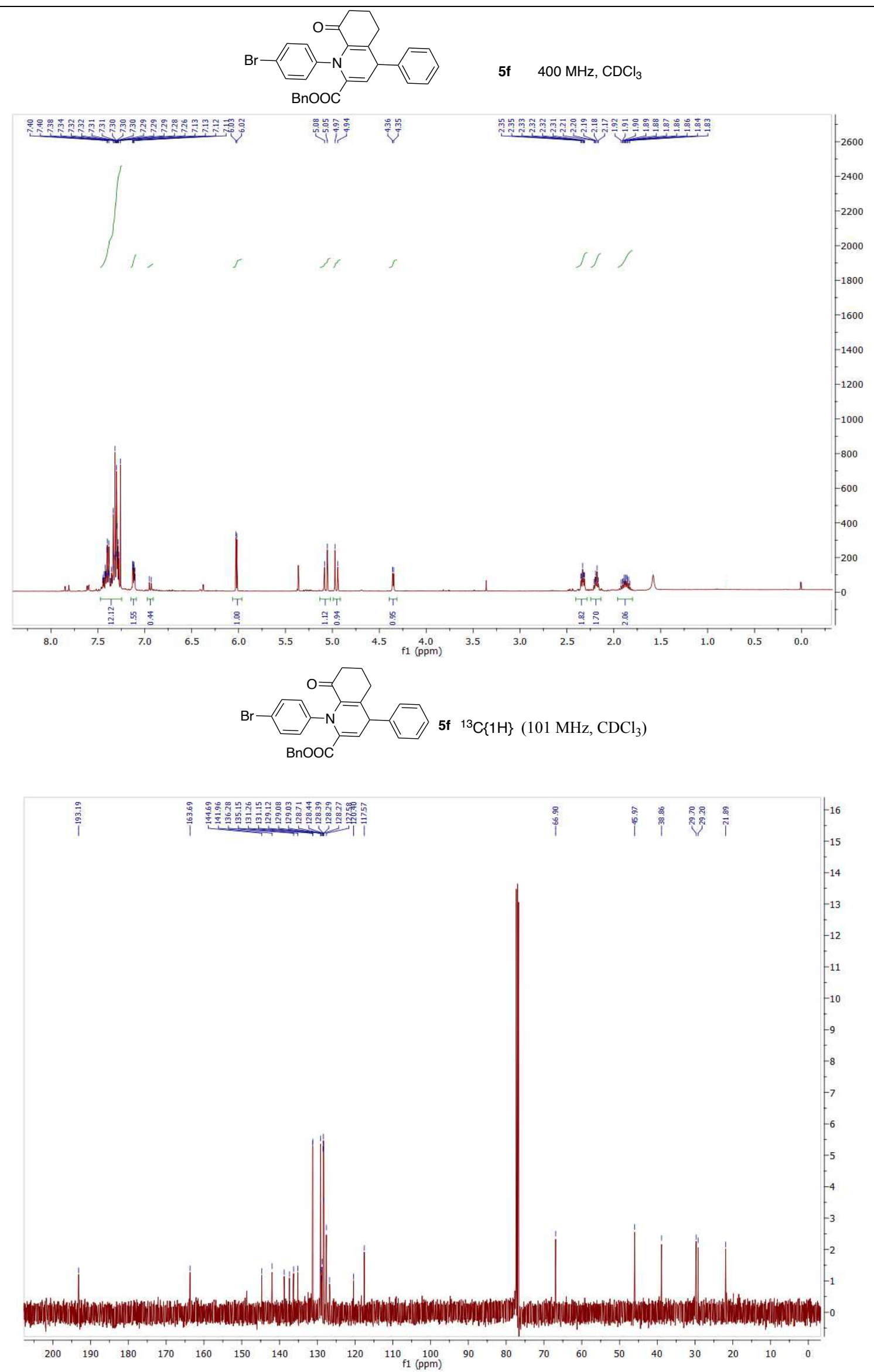


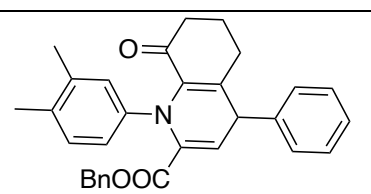

5g $400 \mathrm{MHz}, \mathrm{CDCl}_{3}$
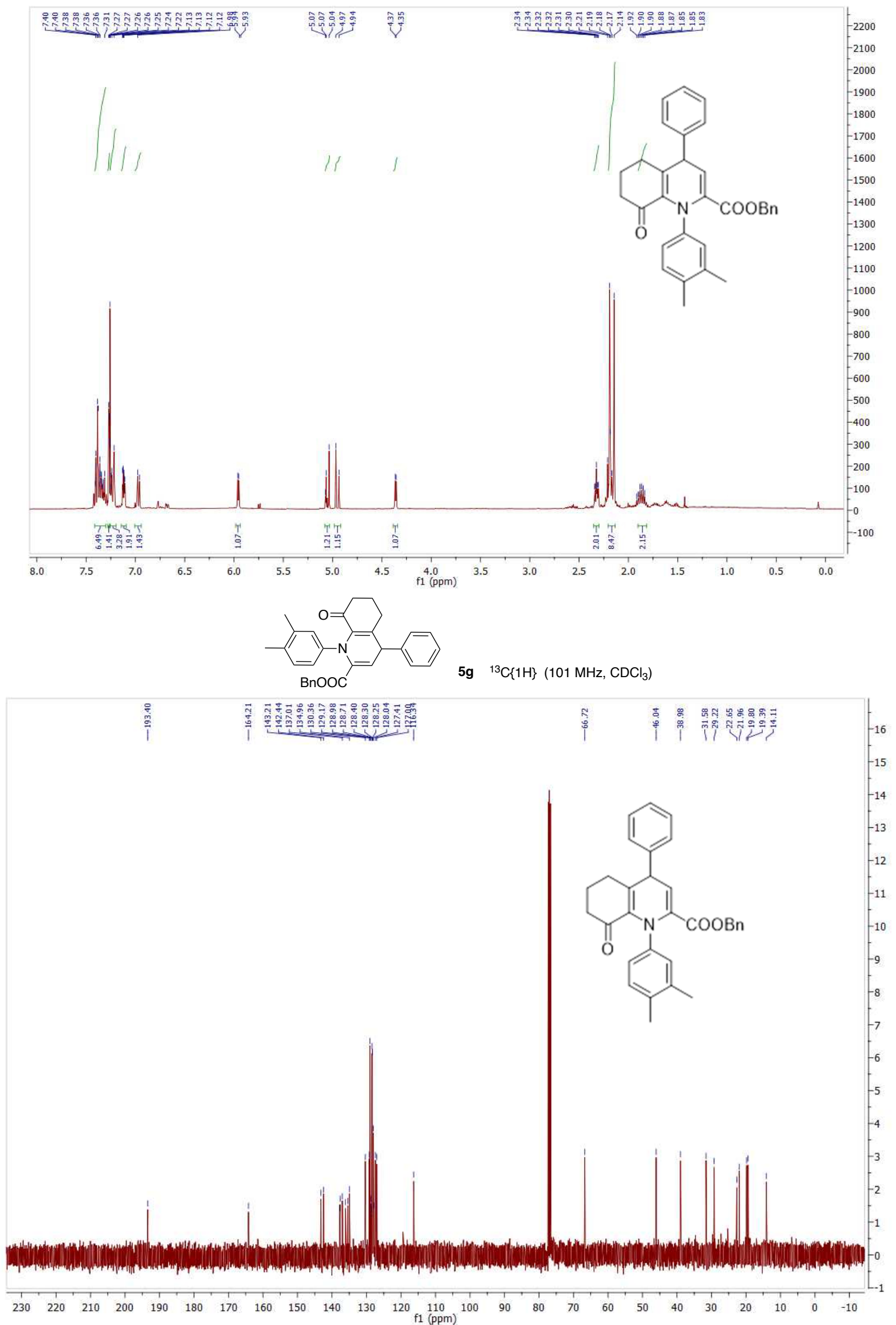


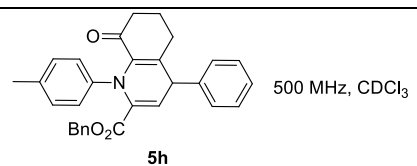

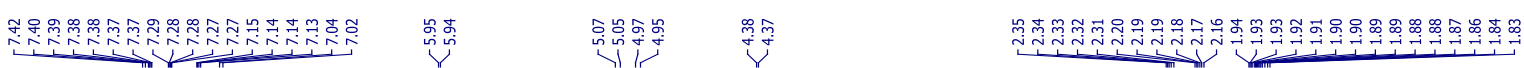
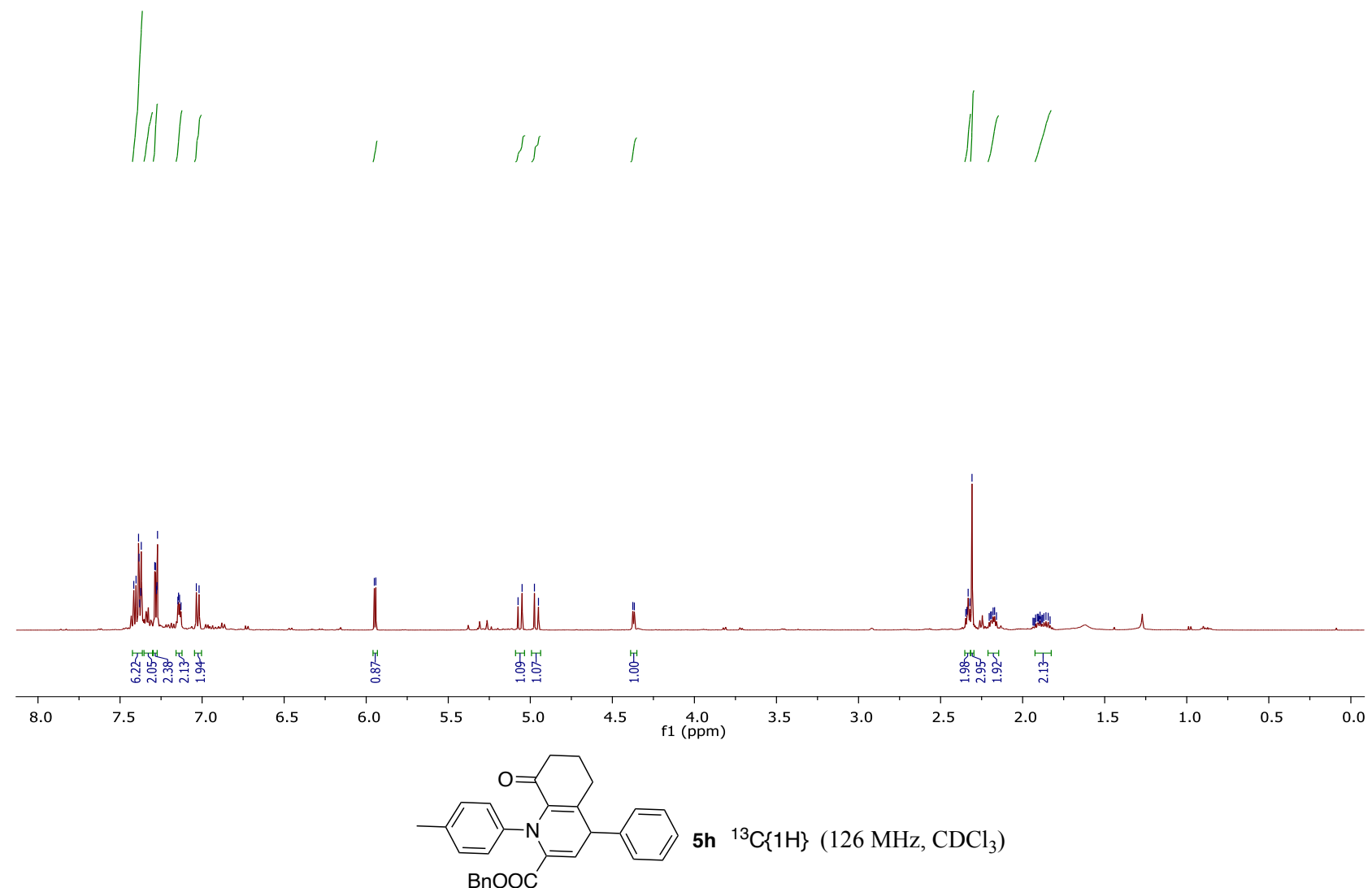

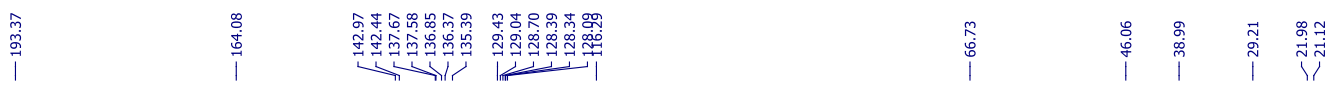

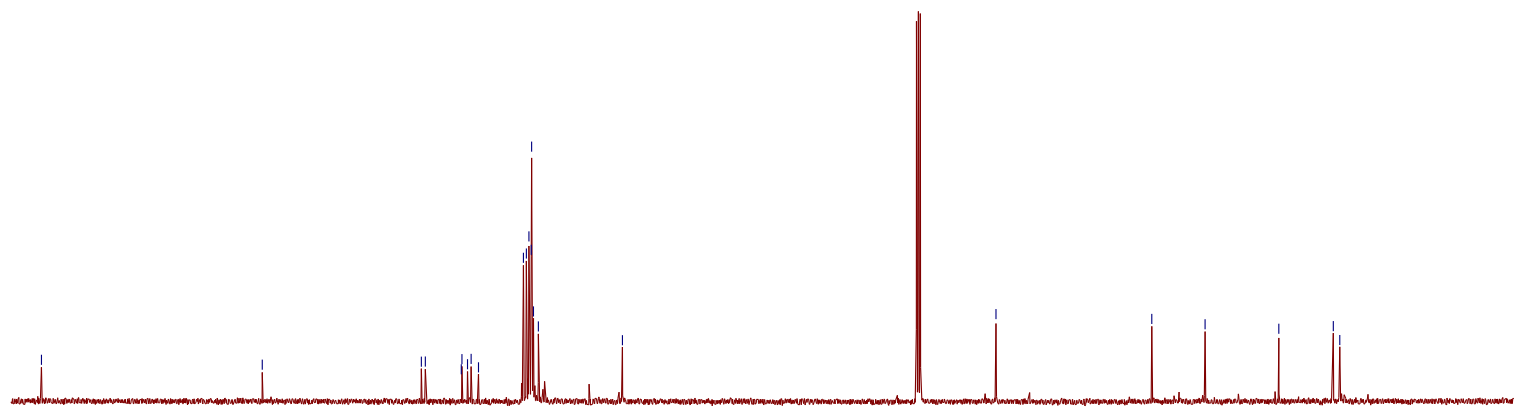

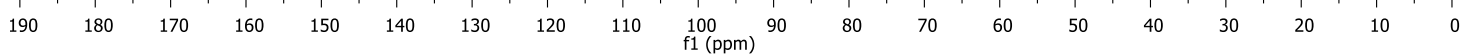




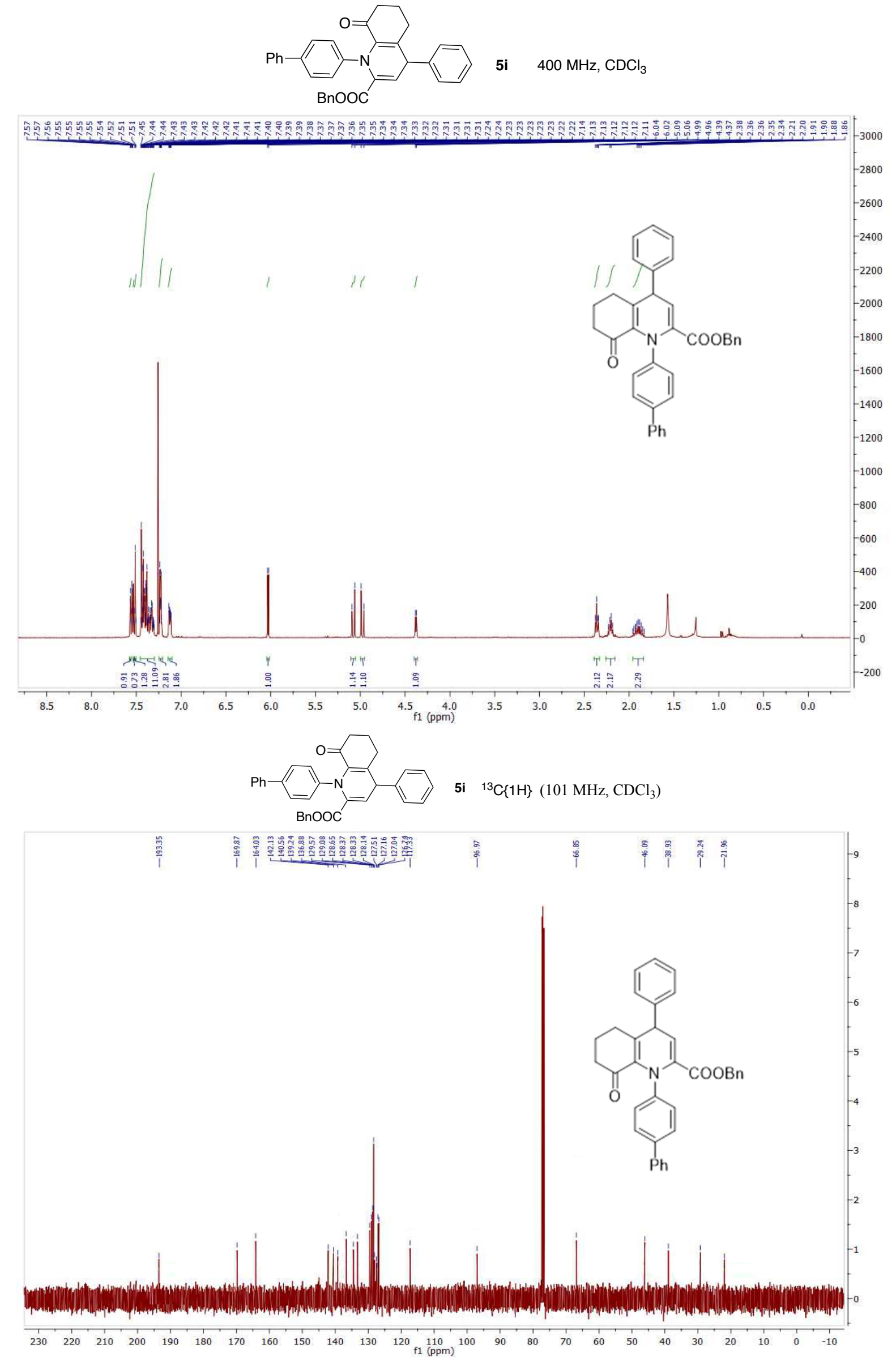



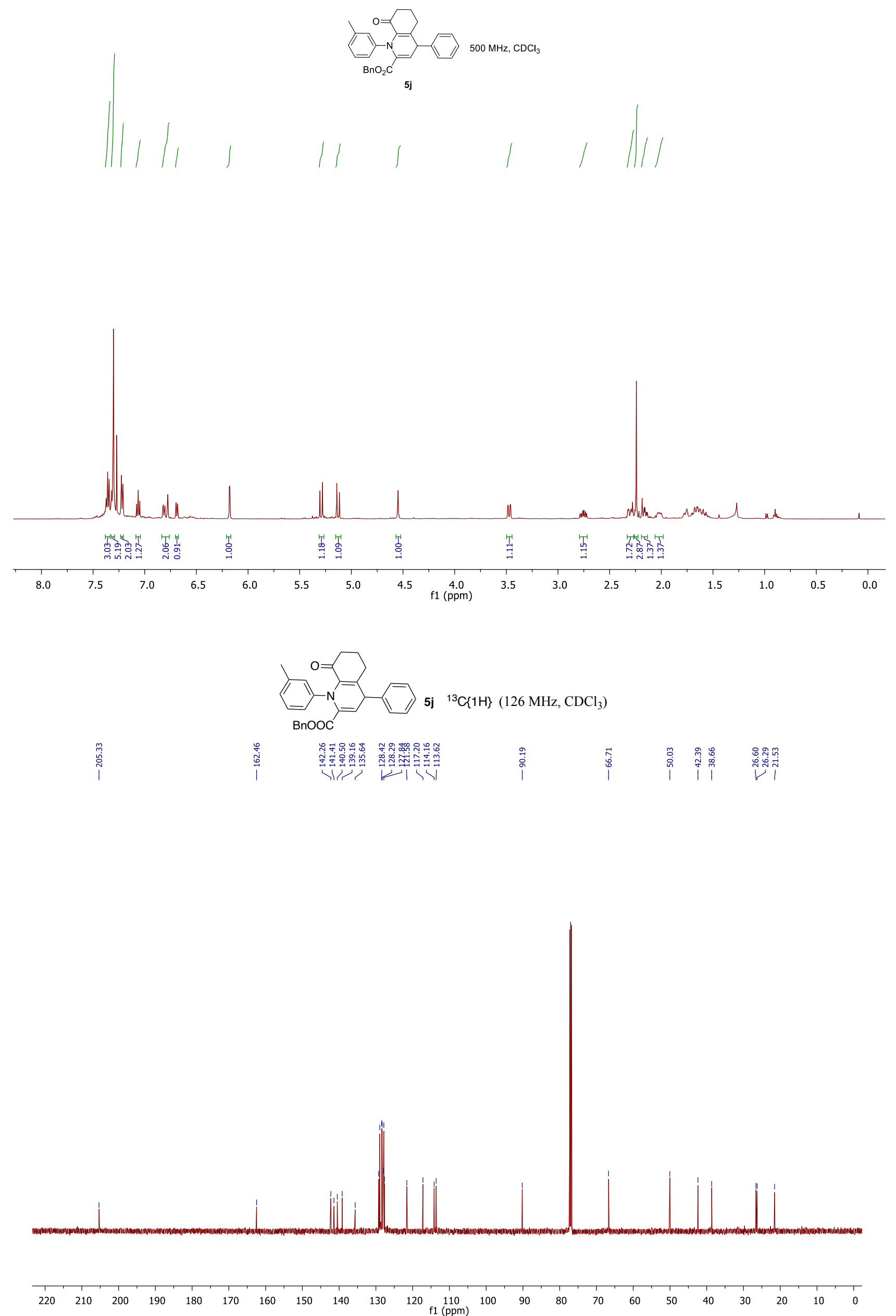